\title{
Identification of thermal material parameters for thermo- mechanically coupled material models
}

\author{
Verification and model dependency
}

\author{
L. Rose $(\mathbb{D} \cdot$ A. Menzel
}

Received: 8 June 2020/ Accepted: 28 October 2020/Published online: 18 January 2021

(C) The Author(s) 2021

\begin{abstract}
The possibility of accurately identifying thermal material parameters on the basis of a simple tension test is presented, using a parameter identification framework for thermo-mechanically coupled material models on the basis of full field displacement and temperature field measurements. Main objective is to show the impact of the material model formulation on the results of such an identification with respect to accuracy and uniqueness of the result. To do so, and as a proof of concept, the data of two different experiments is used. One experiment including cooling of the specimen, due to ambient temperature, and one without specimen cooling. The main constitutive relations of two basic material models are summarised (associated and non-associated plasticity), whereas both models are extended so as to introduce an additional material parameter for the thermodynamically consistent scaling of dissipated energy. The chosen models are subjected to two parameter identifications each, using the data of either experiment and focusing on the determination of thermal material parameters. The influence of the predicted dissipated energy of the models on the identification process is
\end{abstract}

L. Rose $(\bowtie) \cdot$ A. Menzel

Institute of Mechanics, TU Dortmund, Leonhard-Euler-

Str. 5, Dortmund 44227, Germany

e-mail: lars.rose@tu-dortmund.de

\section{A. Menzel}

Division of Solid Mechanics, Lund University, P.O.

Box 118, Lund 221 00, Sweden investigated showing that a specific material model formulation must be chosen carefully. The material model with associated evolution equations used within this work does neither allow a unique identification result, nor is any of the solutions for the underlying material parameters close to literature values. In contrast to that, a stable, that is locally unique, reidentification of the literature values is possible for the boundary problem at hand if the model with nonassociated evolution equation is used and if cooling is included in the experimental data.

Keywords Parameter identification - Thermomechanically coupled problem - Displacement field . Temperature field $\cdot$ Model dependency $\cdot$ Dissipation

\section{Introduction}

Accurate material parameters are the basis of every predictive simulation. The optimal values of these parameters are usually determined by means of a parameter identification. Optimal parameters, however, are not useful if the chosen material model itself is not able to represent important key features of the material behaviour under consideration. If, for example, an isotropic yield surface is used to predict the material response of a strongly anisotropic material undergoing inhomogeneous stress states, the 
computed results will not reflect the real material behaviour, regardless whether the parameters are optimal or not. Likewise, insufficient experimental data may allow a good fit of a model, but the fitted parameters may not represent any other type of boundary value problem due to overfitting.

Although it may be obvious as to what kind of models should be avoided, it is not always clear how certain aspects of a model must be chosen exactly. Regarding the example from before, it is not intuitively clear what kind of anisotropic yield surface fits best to what kind of material, seeing that there are various different formulations established. This knowledge has to be acquired by trial and error, comparing model predictions and experiments. One modelling aspect that has been subject to scientific discussion for almost a century is the question of how to model dissipation within thermo-plastic material models. No final answer exists and it will therefore be the focus of this work to compare two thermodynamically consistent model formulations which differ only in the amount of predicted dissipation in the context of a parameter identification. More precisely speaking, it is the intention of this work to show the impact of two different material models on the result of a parameter identification for thermo-mechanically coupled material models and, furthermore, to show what kind of experimental data is necessary to ensure a stable identification process. This work is a follow-up of a previous publication [22] and addresses further research topics such as model and experiment dependencies in the context of a parameter identification. Only brief summaries are given regarding the framework of the identification, experimental setup as well as the description of the underlying boundary value problem. The reader is referred to the afore mentioned publication for more detailed descriptions.

One of the first reports about the correlation of cold work and heat was published in 1925 by Farren and Taylor, see [5]. Their measurements during dynamic tension tests suggested that a near constant fraction of the cold work was transformed to heat and that the remainder was stored as latent energy in the material. Some time later, Taylor and Quinney reasoned that this finding could not be true for larger strain states, since the latent energy of a material should saturate, see [24]. Within that work, experiments with a twisted rod underlined this line of thought, showing that the dissipated energy depends on the current load state.
This led to the introduction of the so-called TaylorQuinney factor which describes the ratio of cold work to dissipated energy. In later years, a similar factor was introduced to describe the ratio between the rate of cold work, i.e. the stress power, and the rate of dissipated energy, see e.g. [15] and [20]. The latter work also clearly differs between the two aforementioned factors, which were usually denoted by the same symbol albeit describing fundamentally different ratios. At that point, the idea of a strain and strain rate dependent ratio between cold work and dissipated energy was well established, and many further scientific contributions can be found with the focus on the determination of this dependency. Whereas some researchers mainly seek to determine the TaylorQuinney coefficient experimentally, e.g. in [7, 12] using a Kolsky (Split-Hopkinson) pressure bar, or in [9] under uniaxial tension, others place a stronger emphasis on thermodynamical consistency. It has often been stated, that the simple replacement of the predicted dissipation of a model with a fraction of the plastic stress power does not necessarily fulfil the first law of thermodynamics, so that a thermodynamically consistent model which predicts the dissipated energy accurately should be used instead. Hence, several modelling approaches have been proposed and their predictions have usually been compared to experimental data of a dynamic Kolsky (Split-Hopkinson) bar test assuming adiabatic conditions, see e.g. $[21,23,25]$ and [2]. In the latter work, an additional material parameter is introduced by making an extension to the postulated Helmholtz free energy function. This parameter determines how much energy is stored in the material and how much is dissipated during plastic deformations. Thus, it allows a better adaptation of the model to real material behaviour while still satisfying the laws of thermodynamics.

Although the overview above is just a brief excerpt and many more publications could be mentioned, attention is drawn to only two more approaches to the identification of plasticity induced dissipation. In [19] a slightly different concept is presented which tries to avoid the test of all kind of possible energy formulations and rather aims at deducing an appropriate Helmholtz free energy function directly from experimental data. The approach is tested on data of a tensile test assumed to be adiabatic and compares two fundamentally different plasticity model approaches, i.e. associated and non-associated plasticity. The 
results show that the associated format is not able to correctly predict the dissipation obtained from the experiment. The second approach can be found in [18] where an inverse analysis with full field displacement and temperature measurements of an inhomogeneous boundary value problem (BVP) is used to fit the mechanical parameters and the evolution of a TaylorQuinney factor of a simple, not necessarily thermodynamically consistent model to an observed temperature field. A similar method was used in [22] to fit the mechanical as well as the thermal material parameters of a potential based material model to the experimental full field displacement and temperature data of a tensile test under monotonic loading. Apart from differences in the handling of experimental data and the employed material model, the main distinction in these two works is the set of parameters which is optimised. Whereas heat capacity, thermal conductivity and thermal expansion were taken from literature in [18], these parameters were part of the optimisation in [22]. It was therefore shown in the latter work that a stable identification of the thermal material parameters is possible on the basis of a simple tension test.

Regarding the identification of thermal material parameters such as the heat capacity, there are mainly two different approaches to obtain these parameters of a material. The first is the use of calorimetry, necessitating special experimental devices which can be used almost exclusively for that purpose. An alternative was first introduced in [17], the so-called flash-method, where a specimen is subjected to a short laser impulse measuring the thermal response of the material close to the laser impact. This approach was further refined e.g. in [10] and [1] to include an inverse analysis similar to the approach of the Finite-ElementModel-Updating (FEMU) method.

Most of the literature above is either concerned with a precise prediction of dissipated energy, aiming at an equally precise prediction of temperature evolution but taking thermal parameters from literature, or it is concerned with the identification of thermal material parameters leaving aside all coupling effects. In [22], however, a scheme can be found which combines both aspects. The framework presented therein can be used to identify all material parameters of a thermo-mechanically coupled material model, including the thermal ones, under any kind of mechanical load. While the aforementioned publication shows that a stable optimisation is possible on the basis of a specific example (simple tension test with monotonous loading, associated evolution equations without possibility to scale dissipation independently), it also states that the resulting, optimal parameters may vary for different material model formulations. The thermal parameters identified in [22] lead to a very precise fit of the measured temperature field, and the model of that publication was therefore able to represent the plastic heating of the material. The value of the optimal thermal material parameters however, differed significantly from literature values and it can be assumed that they are therefore unable to also represent the cooling of the specimen.

The model formulation is therefore vitally important for the identification of material parameters which can be used to accurately predict the material response of different BVPs, and the cited literature provides information on sensible model formulations. For this work, two thermodynamically consistent material models, either standard associated or non-associated plasticity, are extended to allow a greater flexibility in the predicted dissipation, following the modelling approach presented in [2]. These models are then subjected to a parameter identification for thermomechanically material models by using the framework presented in [22]. That is to say, two different experiments are used as the basis of the identifications to see what kind of data is necessary to obtain a (locally) unique solution and to investigate if monotonous loading (as is used in almost all of the publications mentioned above) holds enough information to find a parameter set which can also represent several loading scenarios, i.e. plastic heating and conduction based cooling of the specimen. The aim of this work is not to compute reliable parameter values which can be used for predictive simulations. This would require the consideration of more than two experiments as in case of this work. Aim of this work is to show that the model formulation has a nonneglectable influence on the numerical value of the optimal material parameters and that a stable identification is possible, albeit adding an additional unknown, i.e. a further material parameter which scales dissipation.

To do so, this article is structured as follows. The essentials of the underlying identification framework for coupled models are summarised in Sect. 2. It is followed by information on the experimental setup in Sect. 3, including a description of the specimens that 
are used for two different tensile tests (monotonous loading and loading-unloading-cooling) as well as a representation of the obtained experimental data. From this experimental data, two material models are motivated in Sect. 4 which are able to represent observed key features. Two different modelling approaches are chosen, whereas one model uses associated evolution equations and the second model uses non-associated evolution equations. The Helmholtz free energy function of both models is extended to account for a material parameter which has an influence on the amount of latent energy during plastic deformations, c.f. [2]. It is worth noting that both models predict the same mechanical material behaviour and only differ in the amount of dissipated energy. All material parameters are analysed with respect to possible correlations. Section 5 provides information on the specific identifications that are performed, starting with a definition of the discretised BVP. The parameters of both models are fitted to the experimental data of either of the two experiments presented before to see what kind of data is necessary for a (locally) unique result that can represent loading and cooling alike. The obtained results are presented and analysed with respect to the remaining error, possible correlations, as well as their distance to literature values. At last, a short discussion can be found in Sect. 6, summing up the main results of this work.

\section{Parameter identification framework}

A Finite-Element-Model-Updating (FEMU) method is used for the parameter identifications within this work. The general concept of this approach can be found in numerous publications and a comprehensive description of the specific framework that was used for this work can be found in [22]. Thus, the framework of a FEMU method is considered to be state of the art and only the essentials of the extension for the handling of temperature data is summarised within this section.

Interpolation An interpolation is usually required in order to compare experimental and simulated data at the same points in space. Experimental displacement data is interpolated on the referential coordinates of the FE mesh in a pre-processing step. Thus, a set of displacements describes the movement of each node throughout the experiment. In contrast to the displacement data, temperature data is only available in the current configuration of the experiment. The experimental displacement sets are therefore used to interpolate the experimental temperature in the current configuration on the position which each node possessed during the experiment at the respective time step.

Objective function For the sake of completeness, the objective function used is specified, i.e.

$$
\begin{aligned}
f= & \sum_{i \in\{x, y, z\}} W_{i}^{\mathrm{u}} \sum_{t=1}^{n_{\mathrm{ts}}}\left[\Delta \mathbf{u}_{i}^{\exp }-\Delta \mathbf{u}_{i}(\boldsymbol{\kappa})\right]_{t}^{2} \\
& +W^{\theta} \sum_{t=1}^{n_{\mathrm{ts}}}\left[\Delta \boldsymbol{\theta}^{\exp }-\Delta \boldsymbol{\theta}(\boldsymbol{\kappa})\right]_{t}^{2} \\
= & f^{\mathrm{u}}+f^{\theta},
\end{aligned}
$$

cf. [22]. It combines the error in relative displacements and the error in temperature rise. The weighting factors $W$ are used to avoid a clash of units and to ensure an equal contribution of each of the different error contributions. It is worth noting that the use of relative displacements $\Delta \mathbf{u}$ compensates unwanted rigid body motions which would otherwise have a strong influence on the results of the identification process, if not treated otherwise. The objective function can furthermore be extended to include additional measurable quantities, such as the integral reaction force, see [14] or [13].

\section{Experiments}

Information about the experiments which are used for the parameter identification process is given within this section. The type of specimen which is chosen for the experiments as well as the experimental setup are briefly introduced first. A more detailed description can be found in [22] where the same setup was used. Since two types of experiments are performed, the different load types are explained, introducing an experiment with and one without the cooling down phase of the specimen. Data of the former experiment was also already used in [22] whereas data of the latter experiment marks a distinct extension of the work at hand. Afterwards, the measured displacement field and temperature field data is visualised and analysed. Only one experiment per testing condition is later used within this proof of concept. A real parameter 
identification should be based on a statistically relevant number of experiments, if its result shall be used for predictive simulations.

\subsection{Specimens}

The experiments were performed with flat tensile test specimens made from the aluminium alloy AW6016. All specimens are cut from a sheet metal plate such that the tensile direction aligns with the rolling direction of the plate. Figure 1 displays the chosen geometry which is based on the recommendations for tensile tests as defined in DIN 50125.

\subsection{Experimental setup}

Each specimen is observed by means of a DIC as well as by a thermography system throughout the whole experiment. Thus, displacement field and temperature field are measured parallelly to be later used for the identification process. Regarding the mechanical load, all experiments are controlled by an electro-mechanical tension machine. DIC and thermography system are placed on opposite sides of the specimen to avoid erroneous temperature measurements, see [22] for further details. All systems are started simultaneously by means of a trigger signal but may save corresponding data at different rates.

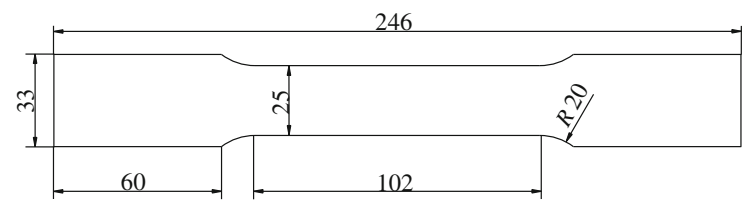

(a) Technical drawing - top view.

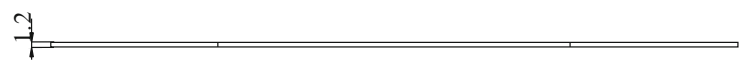

(b) Technical drawing - front view.

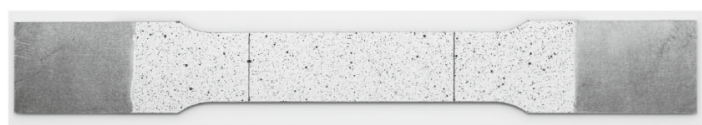

(c) Stochastic pattern for DIC system on topside of specimen.

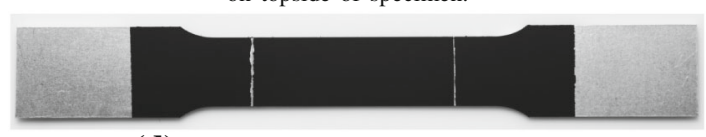

(d) Black paint for thermography system on backside of specimen.

Fig. 1 Dogbone specimen for tensile testing. Reprinted from [22], with permission from Elsevier
All experiments are subjected to the same displacement controlled loading path with a constant cross head speed of $0.14 \mathrm{~mm} / \mathrm{s}$ over a time interval of $140 \mathrm{~s}$. Some measurements were stopped right after this loading phase and some were continued to also include an unloading and cooling towards room temperature of the specimen. More precisely speaking, the respective specimens are unloaded displacement controlled until the force reaches a value of $0 \mathrm{~N}$. The unloading takes about $3 \mathrm{~s}$ and the specimen is observed until a total time of 10 minutes has passed, see Fig. 2. The first type of experiment will henceforth be referred to as "without cooling" and the second type as "with cooling".

\subsection{Experimental data}

Although full field measurements are made, the experimental stress strain relation can be computed on the basis of the obtained displacement field and force signal. Assuming a homogeneous distribution of stress and strain within the area of interest, the associated component of the Piola stress tensor and of the deformation gradient in tensile direction, $P_{\|}$and $F_{\|}$respectively, allow a direct visualisation and interpretation of the experimental data. Figure 3 shows that both experiments have an almost identical stress strain relation starting with linear elastic material behaviour followed by plasticity with a saturation type of hardening. Neither experiment shows indications of necking.

Due to the boundary conditions which are displayed in Table 1, the temperature field is inhomogeneously distributed throughout the experiment with its extremum in the centre of the specimen, see Fig. 4.

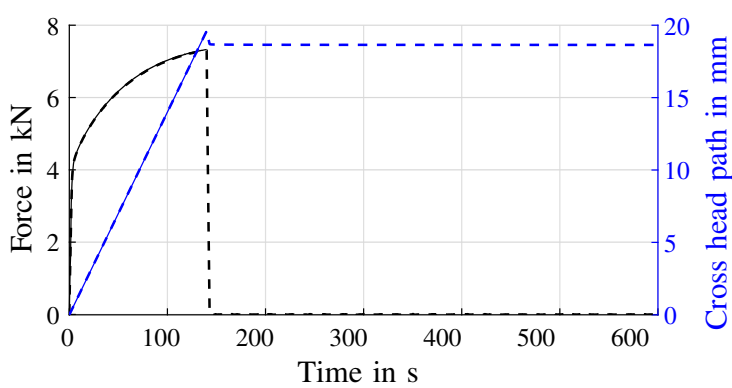

Fig. 2 Loading path for the experiments, with cooling (- -) and without cooling (-) 


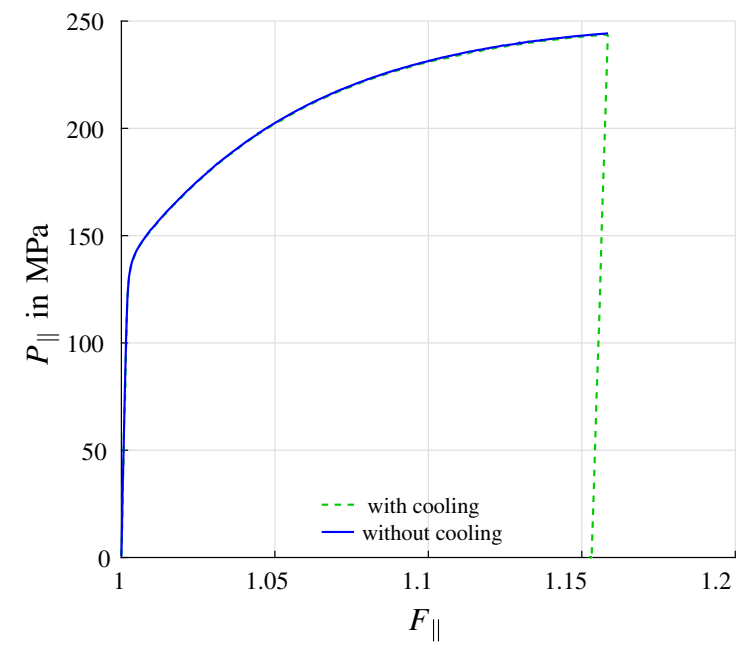

Fig. 3 Stress-strain relation of the experiments

Table 1 Measured temperatures at the boundaries of the specimen

\begin{tabular}{lll}
\hline Experiment & Clamping jaws $\left[{ }^{\circ} \mathrm{C}\right]$ & Air $\left[{ }^{\circ} \mathrm{C}\right]$ \\
\hline With cooling & 23.80 & 24.50 \\
Without cooling & 23.10 & 24.25 \\
\hline
\end{tabular}

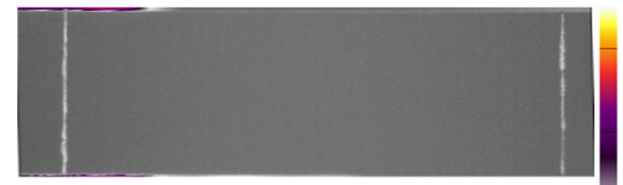

(a) Initial temperature distribution.

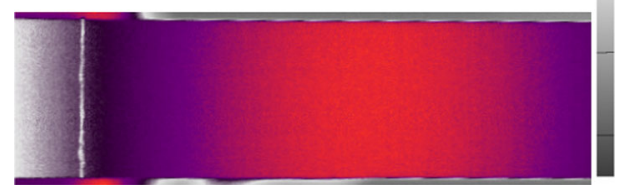

$24.0^{\circ} \mathrm{C}$

(b) Temperature field at $140 \mathrm{~s}$.

Fig. 4 Temperature field of specimen during the experiment

The temperature of the centre point of the specimen over time is given in Fig. 5, since the evolution of a local quantity over time can be visualised and interpreted straightforwardly.

It is worth mentioning that, although the thermography system only measures the temperature of areas which are fixed in space, the simultaneously obtained displacement data is used to find the temperature at the current location of the centre point. Such an algorithm can for example be found in [22] and [11]. The data of Fig. 5 indicates that each experiment considered here undergoes some elastic cooling before plastic heating leads to a temperature rise of approximately $3 \mathrm{~K}$. The experiment with cooling furthermore shows a rise in temperature during elastic unloading, followed by the expected cooling down of the specimen.

Figure 5 furthermore highlights the reproducibility as the two experiments were performed with specimens from the same batch but several months apart.

\section{Material models}

The choice of a specific model can have a significant influence on the result of a parameter identification. Hence, two different material models are introduced within this section to be later compared in the context of parameter identification. The underlying material parameters are briefly analysed with respect to identifiability on the basis of the experimental data at hand.

\subsection{Model formulation}

The dissipated energy during plastic material deformations is the main reason for the heating of the specimen considered here. Thus, and for the sake of comparison, the main assumptions and equations necessary for the derivation of two material models are presented which differ qualitatively in the amount of dissipated energy. The first material model uses associated evolution equations and includes the Gough-Joule-Effect as well as plastic heating. It features isotropic elasticity, a Hill-type yield surface and exponential hardening. The second material model uses non-associated evolution equations. It also includes the Gough-Joule-Effect and plastic heating as well as isotropic elasticity, a Hill-type yield surface and a saturation type of isotropic hardening. Furthermore, a factor $\beta$ scaling single dissipation contributions is included which does not influence the thermodynamic consistency of either model as is proposed in [2]. Heat exchange with the environment is considered by means of surface elements for either model.

The models will show a similar mechanical behaviour, yet differ in the prediction of dissipated energy. Furthermore, the aforementioned factor $\beta$ can be used to scale the amount of dissipated energy which 
Fig. 5 Stress, strain and temperature evolution over time for the two experiments, with cooling (- -) and without cooling (-)

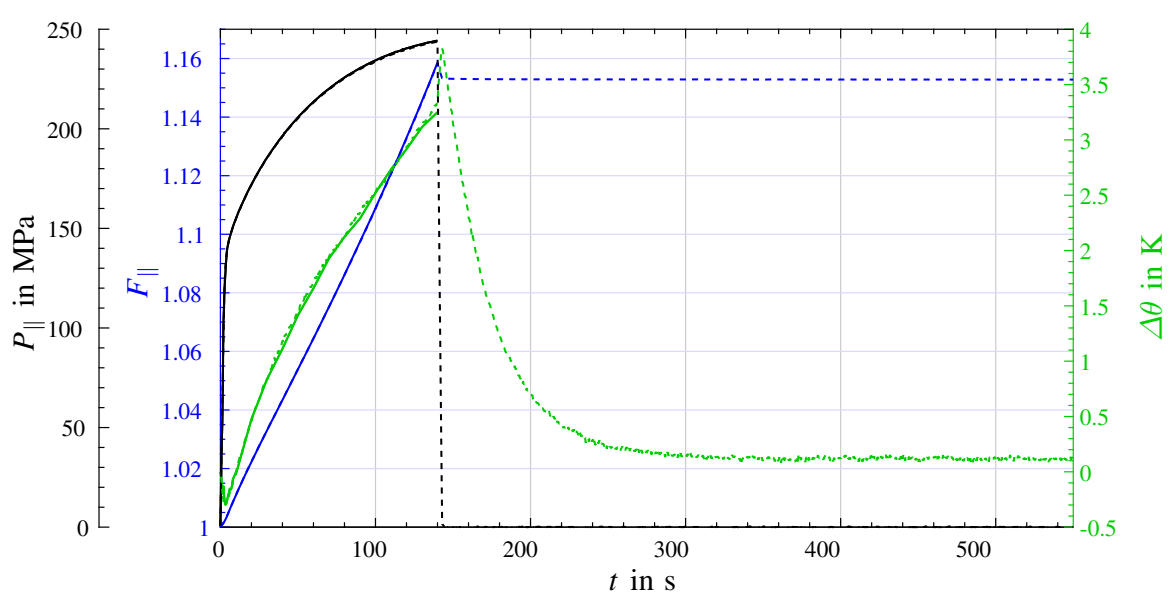

would otherwise solely depend on mechanical material parameters. It is worth noting that $\beta$, as introduced in [2] and as used within this work, is not a TaylorQuinney factor. In general, it describes neither the ratio of plastic to dissipated work nor the ratio of plastic power to dissipated energy. It is merely an additional material parameter, describing the amount of energy which is for example necessary for the creation and upholding of dislocations, the so-called stored or latent energy of cold work.

Both material models are set up in a finite deformation setting with the deformation gradient $\boldsymbol{F}=\nabla_{X} \boldsymbol{\varphi}$ of the deformation mapping $\boldsymbol{x}=\boldsymbol{\varphi}(\boldsymbol{X}, t)$. We shall assume a multiplicative split into an elastic contribution and a plastic part, i.e. $\boldsymbol{F}=\boldsymbol{F}^{\mathrm{el}} \cdot \boldsymbol{F}^{\mathrm{pl}}$. The free energy function

$$
\begin{aligned}
& \psi=\psi^{\mathrm{el}}+\psi^{\mathrm{pl}}+\psi^{\text {ther }}+\psi^{\text {coup }}+\psi^{\mathrm{ns}} \quad \text { with } \\
& \psi^{\mathrm{el}}=\frac{\lambda}{2} \ln ^{2}\left(J^{\mathrm{el}}\right)+\frac{\mu}{2}\left[\operatorname{tr}\left(\boldsymbol{C}^{\mathrm{el}}\right)-3\right]-\mu \ln \left(J^{\mathrm{el}}\right), \\
& \psi^{\text {ther }}=c_{0}\left[\theta-\theta_{0}-\theta \ln \left(\frac{\theta}{\theta_{0}}\right)\right], \\
& \psi^{\text {coup }}=-3 \alpha_{\mathrm{exp}} K_{\text {bulk }}\left[\theta-\theta_{0}\right] \frac{\ln \left(J^{\mathrm{el}}\right)}{J^{\mathrm{el}}}, \\
& \psi^{\mathrm{ns}}=[1-\beta] M_{y_{0}} \alpha,
\end{aligned}
$$

is postulated for both models, using the determinant of the elastic deformation gradient $J^{\mathrm{el}}=\operatorname{det}\left(\boldsymbol{F}^{\mathrm{el}}\right)$, the right, elastic Cauchy-Green tensor $\boldsymbol{C}^{\mathrm{el}}=\left[\boldsymbol{F}^{\mathrm{el}}\right]^{\mathrm{t}} \cdot \boldsymbol{F}^{\mathrm{el}}$, as well as the internal hardening variable $\alpha$ and the absolute temperature field $\theta(\boldsymbol{X}, t)$. A non-standard contribution is added, as is proposed in [2], which will turn out to control the amount of energy which is either dissipated or latent during plasticity evolution. The only difference in the two free energy formulations lies within the chosen plastic part,

Model $1 \quad \psi^{\mathrm{pl}}=\left[M_{y_{\infty}}-M_{y_{0}}\right]\left[\alpha+\frac{\exp \left(-H_{\exp } \alpha\right)}{H_{\exp }}\right]$

Model $2 \quad \psi^{\mathrm{pl}}=\frac{1}{2} h \alpha^{2}$.

Regarding the derivation of the required constitutive equations, the local form of the Clausius-Duhem inequality can be used to obtain the constitutive equations for the Piola stress tensor $\boldsymbol{P}$, driving force $K$ for the internal hardening variable and, based on $\boldsymbol{P}$, for the Mandel stress tensor $\boldsymbol{M}$, i.e.

$$
\begin{aligned}
& \boldsymbol{P}=\frac{\partial \psi}{\partial \boldsymbol{F}^{\mathrm{el}}} \cdot\left[\boldsymbol{F}^{\mathrm{pl}}\right]^{-\mathrm{t}}, \\
& K=-\frac{\partial \psi}{\partial \alpha}, \\
& \boldsymbol{M}=\left[\boldsymbol{F}^{\mathrm{el}}\right]^{\mathrm{t}} \cdot \boldsymbol{P} \cdot\left[\boldsymbol{F}^{\mathrm{pl}}\right]^{\mathrm{t}}=\left[\boldsymbol{F}^{\mathrm{el}}\right]^{\mathrm{t}} \cdot \frac{\partial \psi}{\partial \boldsymbol{F}^{\mathrm{el}}}
\end{aligned}
$$

by following the steps of the Coleman and Noll procedure. The driving force of the internal hardening variable is reduced to 


$$
\bar{K}=-\frac{\partial \psi^{\mathrm{pl}}}{\partial \alpha}
$$

for standard models without the non-standard energy contribution, which can also be retrieved by choosing $\beta=1$. Fourier's law

$$
\boldsymbol{q}=-\kappa_{\text {therm }} \nabla_{\boldsymbol{x}} \theta
$$

is assumed to govern the heat flux in the spatial configuration, so that the Fourier part of the ClausiusDuhem inequality is always fulfilled. Regarding heat exchange with the environment, the heat flux normal to the current surface of the body is incorporated by using the equation

$$
q_{0}=\boldsymbol{n} \cdot \boldsymbol{q}=-\alpha_{\mathrm{con}}\left[\theta^{\mathrm{M}}-\theta\right],
$$

depending on the convection or conduction coefficient $\alpha_{\mathrm{con}}$ and on the temperature of the surrounding medium $\theta^{\mathrm{M}}$, see e.g. [3] for more details.

The approach for a standard model formulation is used to define the evolution equations for the plastic velocity gradient $\boldsymbol{L}^{\mathrm{pl}}=\dot{\boldsymbol{F}}^{\mathrm{pl}} \cdot\left[\boldsymbol{F}^{\mathrm{pl}}\right]^{-1}$ and the internal hardening variable $\alpha$, i.e.

$$
\begin{gathered}
\boldsymbol{L}^{\mathrm{pl}}=\lambda_{\mathrm{pm}} \frac{\partial g}{\partial \boldsymbol{M}}, \\
\dot{\alpha}=\lambda_{\mathrm{pm}} \frac{\partial g}{\partial \bar{K}},
\end{gathered}
$$

with the Lagrange or plastic multiplier $\lambda_{\mathrm{pm}}$ and the plastic potential $g$. The plastic potential is chosen differently for each model,

Model $1 \quad g=\Phi$

Model $2 \quad g=\Phi+\frac{1}{2} \frac{b}{h} \bar{K}^{2}$.

Both potentials make use of the yield function

$$
\Phi=\sqrt{\boldsymbol{M}: \mathrm{G}: \boldsymbol{M}}-\beta M_{y_{0}}+K
$$

with the fourth order tensor $G$ representing Hill-type plastic anisotropy. A comment on the reason for the specific formulation of the plastic potential for the non-associated model can be found in Remark 2 at the end of this subsection. If the definition of the driving force $K$ is inserted into the chosen form of the yield function, the standard formulation of a Hill type yield surface

$$
\begin{aligned}
\Phi= & \sqrt{\boldsymbol{M}: \mathrm{G}: \boldsymbol{M}}-M_{y_{0}}+\bar{K}, \\
= & {\left[F\left[M_{11}-M_{22}\right]^{2}+G\left[M_{33}-M_{11}\right]^{2}\right.} \\
& +H\left[M_{22}-M_{33}\right]^{2}+2 L M_{12}^{2}+2 M M_{23}^{2} \\
& \left.+2 N M_{13}^{2}\right]^{\frac{1}{2}}-M_{y_{0}}+\bar{K}
\end{aligned}
$$

is retrieved, introducing the six Hill parameters $F, G$, $H, L, M, N$ with the coefficients of the Mandel stress tensor referring to the underlying (aligned) orthonormal frame. Time integration is done by means of a simple backward Euler scheme.

For the sake of interpretation in later sections, the balance of energy is given as

$$
c_{0} \dot{\theta}=\rho_{0} r-\nabla_{\boldsymbol{X}} \cdot \boldsymbol{Q}+\mathcal{D}^{\mathrm{mech}}+\theta \frac{\partial^{2} \psi}{\partial \boldsymbol{F}^{\mathrm{el}} \partial \theta}: \dot{\boldsymbol{F}^{\mathrm{el}}}
$$

with $\boldsymbol{Q}=\operatorname{det}(\boldsymbol{F}) \boldsymbol{q} \cdot \boldsymbol{F}^{\mathrm{t}}$ and with the referential heat capacity $c_{0}=-\theta \frac{\partial^{2} \psi}{\partial \theta \partial \theta}$ being constant due to the chosen form of the thermal free energy contribution as introduced in (4). Furthermore, the particular models at hand yield fundamentally different equations for the mechanical dissipation,

Model $1 \quad \mathcal{D}^{\text {mech }}=\beta \lambda_{\mathrm{pm}} M_{y_{0}}$

Model $2 \quad \mathcal{D}^{\text {mech }}=\lambda_{\text {pm }}\left[b h \alpha^{2}\right.$

$$
\left.+[1-\beta] b M_{y_{0}} \alpha+\beta M_{y_{0}}\right] \text {. }
$$

Equations (22) and (23) show that the dissipation of both models is always larger or equal to zero as long as $\beta$ lies within the model specific boundaries

Model $1 \quad \beta \geq 0$,

Model $2 \quad 0 \leq \beta \leq 1 \quad \vee \quad \beta_{1} \leq \beta \leq \beta_{\mathrm{u}}$, with

$$
\beta_{\mathrm{u}, 1}=\frac{M_{y_{0}} b+2 h\left[1 \pm \sqrt{\frac{M_{y_{0}} b}{h}+1}\right]}{M_{y_{0}} b} .
$$

A detailed derivation of the limit values of $\beta$ for model 2 can be found in Appendix 1.

Remark 1 Regarding model 1, the constitutive equation for stresses, the evolution equation for the plastic contribution to the deformation gradient and internal hardening variables as well as the yield 
surface are independent of the non-standard addend to the free energy and therefore independent of the parameter $\beta$ which only influences the amount of dissipated energy. The mechanical response of model 1 is therefore identical to the associated standard model formulation, if coupling effects due to the rise of $3 \mathrm{~K}$ for the case at hand are neglected. Furthermore, the chosen evolution equations for model 1 can also be obtained through the postulate of maximum dissipation.

Remark 2 There are general frameworks wellestablished, such as the one of generalised standard material (GSM), which guarantee a non-negative dissipation if the plastic potential of a model with non-associated evolution equations is defined accordingly. The commonly used formulation

$\tilde{g}=\Phi+\frac{1}{2} \frac{b}{h} K^{2}$

meets all requirements of the GSM approach and therefore also guarantees a non-negative dissipation. However, this choice for a plastic potential leads to evolution equations that depend on parameter $\beta$. Thus, the evolution equations are no longer comparable to the ones of a standard model formulation, i.e. without the non-standard energy contribution. This, in our case, undesired effect can be remedied by the simple modification in Equation (19). The alternative formulation may violate some of the conditions of the GSM approach, so that its line of reasoning can not be applied to the second model. It is therefore shown in Appendix 1 that this specific choice nonetheless fulfils the dissipation inequality. The mechanical response of model 2 is therefore identical to the standard model formulation if coupling effects due to the experimentally observed rise of $3 \mathrm{~K}$ are neglected so that parameter $\beta$ only influences the amount of dissipated energy.

Remark 3 The mechanical response of both models is identical to the one of a standard model without the non-standard addend $\psi^{\text {ns }}$ of the free energy, since this addend only influences the amount of dissipated energy. It was shown in [22] for such standard formulations of these two models that the mechanical response is identical if

$b=H_{\exp } \quad$ and
$\frac{h}{b}=\left[M_{y_{\infty}}-M_{y_{0}}\right]$

in the range of small strains assuming a neglectable influence of the low rise in temperature. The mechanical response of the two models at hand is therefore also identical for small strains and can be assumed to be at least comparable for large deformations. Thus, the hardening parameters $H_{\exp }$ and $M_{y_{\infty}}$ will be used for both models in the following sections.

Remark 4 It is worth noting that Young's modulus $E$ and Poisson's ratio $v$ are used within the identification process instead of the two Lamé parameters $\lambda, \mu$ and the bulk modulus $K_{\text {bulk }}$ which were used for the definition of the energy contributions above.

\subsection{Material parameters}

It is important to know which influence each material parameter has on the material response in order to obtain meaningful results through a parameter identification scheme. More precisely speaking, the question of identifiability must be raised and answered with respect to the kind of experiments which are required for the model at hand. This can be done either by means of the sensitivity of the objective function w.r.t. the material parameters, i.e. the Jacobian matrix of the objective function evaluated at the solution point of the identification, see [6], by an analysis of the underlying constitutive equations, or by using a grid search method in order to receive information towards the uniqueness of the solution within a certain area in parameter space.

The last two options are considered within this work, starting with an analysis of the constitutive equations to see which of the 15 material parameters from Table 2 are correlated and which are not. This analysis provides information as to which parameters of the models can and which can not be identified on the basis of the experiments at hand, information on the correlation of material parameters and information on the possibility to identify subsets independently. Mechanical parameters which are found to have no influence on the boundary value problem at hand are directly excluded from the identification process. Different and suitable experiments should generally be used for such cases, but it is not the aim of this work to obtain mechanical material parameters which can be used for predictive simulations of different 
Table 2 Material parameters of the models

\begin{tabular}{ll}
\hline$E$ & Young's modulus \\
$v$ & Poissons ratio \\
$M_{y_{0}}$ & Initial yield limit \\
$F, G, H, L, M, N$ & Hill parameters \\
$M_{y_{\infty}}$ & Exponential hardening limit \\
$H_{\exp }$ & Exponential saturation speed \\
$\alpha_{\exp }$ & Thermal expansion coefficient \\
$\kappa_{\text {therm }}$ & Thermal conduction coefficient \\
$c_{0}$ & Heat capacity \\
$\beta$ & Dissipation factor \\
\hline
\end{tabular}

boundary value problems. Rather, the identification of suitable thermal material parameters which can be used for different load scenarios is investigated and the influence of certain parts within the model formulations are to be underlined.

The mechanical response of both models considered is almost identical and the predicted mechanical response does not differ from the standard formulation of either model for a low rise in temperature.

There is no significant correlation between the two elastic material parameters $E$ and $v$ if displacements in tension and lateral direction are used. Furthermore, these parameters can be identified separately in a first step, since the material response at the start of the experiments is unaffected by the plastic material parameters and is also assumed to be unaffected by the low rise in temperature and hence unaffected by the thermal material parameters. Regarding the plastic material parameters $M_{y_{0}}, M_{y_{\infty}}, H_{\text {exp }}, F, G, H, L, M$ and $N$, all (apart from $F, G$ and $H$ ) have a unique interpretation so that no significant correlation is to be expected. However, $L, M$ and $N$ have no effect on the simulation results of the simple tension test considered since these are related to shear components of the Mandel stress tensor. Of the three Hill parameters related to normal stress components $F, G$ and $H$, only one can be identified by means of data from a simple tension test. It was discussed in [22] that the two remaining parameters can be randomly prescribed without affecting the identification result. Regarding the subdivision of the identification process, the assumption that the small increase in temperature has no significant effect on the mechanical response allows the identification of the plastic material parameters after the elastic parameters and prior to the fit of the thermal material parameters $\alpha_{\exp }$, $\kappa_{\text {therm }}, c_{0}$ and $\beta$. As presented in [22], the standard formulation of the model which is reproducible by choosing $\beta=1$ allows a stable identification of the three parameters $\alpha_{\text {exp }}, \kappa_{\text {therm }}$ and $c_{0}$ on the basis of a simple tension test without cooling. This indicates that there is no significant correlation between these three thermal material parameters for that specific inverse problem. In the case of the non-standard model formulation, however, parameter $\beta$ can have a high correlation to the remaining thermal material parameters, depending on the chosen BVP. The parameter is not redundant, but its unique identification greatly depends on the thermal boundary conditions as well as on the precise influence of $\beta$ on the dissipation. A general statement about the correlation between the four thermal parameters of the models at hand therefore proves to be difficult, but an interpretation is given in Sects. 5.2.3 and 5.3.3, where the thermal boundary conditions for the BVP considered in this work are defined.

In summary, it is therefore sufficient to further investigate the correlation between $\beta$ and the other three thermal material parameters, since the correlation of all other material parameters is already specified and non-critical. This investigation is done by means of a grid search approach during the identification of the thermal material parameters. If several initial guesses lead to the same result, it is a strong indicator that a unique solution exists within a certain area in parameter space. In contrast thereto, the inverse problem is ill-posed in case several starting points lead to different solutions yielding an identical objective function value and an identical material response.

With the two material models at hand, it is now possible to determine which model formulation, i.e. which kind of dissipation, and within each formulation what amount of dissipation represents the observed material behaviour most appropriately. It can also be determined what kind of data is necessary to find a unique solution (within a certain area in parameter space) for all four thermal material parameters. We will furthermore investigate whether it is possible to retrieve thermal parameters which can predict plastic heating as well as purely thermal effects with the data at hand. 


\section{Parameter identification}

The two material models of Sect. 4 are compared in the context of a parameter identification within this section. More precisely speaking, the influence of the different kinds of dissipation behaviour of the two models are of interest when determining which of the two models can predict the mechanical and the thermal material behaviour more accurately. Hence, the two different experiments from Sect. 3 are used to test whether the models are able to represent different loads equally well. The parameter identification process is split into three parts, identifying first the elastic, then the plastic and finally the thermal material parameters as was proposed in [22]. The applied optimisation scheme itself is a gradient-free method from the open source toolbox NLopt [8], as documented in [16] and [4]. At first, however, the boundary value problem as well as the weighting factors $W$ within the objective function are specified.

\subsection{Boundary value problem}

The definition of the boundary value problem (BVP) must represent the experimental setup as closely as possible. The BVP at hand was already described in [22] and differs only in the applied load curves. Hence, only a brief summary is given below and the interested reader is kindly referred to the afore mentioned publication.

\subsubsection{Finite element discretisation}

Only a fourth of the specimen is modelled using the dimensions from Sect. 3.1, taking into account the symmetry of the specimen and of the deformation. Each specimen geometry is measured prior to an experiment, but deviations towards the technical drawing are small enough to be neglected.

3840 linear brick elements are used for the approximation of the displacement and temperature fields together with an additional 1280 surface elements to take into account heat exchange with the environment.

\subsubsection{Boundary conditions}

The boundary conditions include mechanical and thermal parts and must represent the underlying experiments as closely as possible

Mechanical boundary conditions Nodes on the green (left) surface in Fig. 6 are clamped in $y$-direction and the experimental reaction force is applied to the nodes on the blue (right) surface. The simulation is therefore force driven following the experimental reaction force of the related experiment as displayed in Fig. 2. Not the entire loading path is used for all identifications, and the exact part which is prescribed is defined in the related subsections as this work proceeds. Furthermore, symmetry boundary conditions are applied.

Thermal boundary and initial conditions Surface elements are used to incorporate the heat exchange with the environment. The heat exchange through the clamping jaws appears to dominate for the specific experiments at hand. In that sense, the convection coefficients for those elements which model contact to air are set to $\alpha_{\mathrm{con}}^{\mathrm{air}}=0 \mathrm{~W} /\left[\mathrm{m}^{2} \mathrm{~K}\right]$, while the conduction coefficients of the surface elements which model contact to the clamping jaws are set to $\alpha_{\text {con }}^{\text {clamp }}>10^{8} \mathrm{~W} /$ $\left[\mathrm{m}^{2} \mathrm{~K}\right]$. Regarding the initial temperature assigned to each node, the measured temperature of the clamping jaws, see Table 1, is used for all nodes.

Weighting factors The weights $W$ for the error in displacements and temperatures within the objective function (1) are set as follows. Nodes outside the black rectangle $(|y|>20 \mathrm{~mm})$ in Fig. 6a are neglected, as well as nodes which are too close to the edge of the specimen $(x>11 \mathrm{~mm})$. The respective weights are set to zero. The weights for $x$ - and $y$-components of the remaining nodes are chosen so that an almost equal

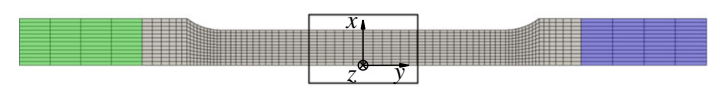

(a) Top view - Nodes on the green (left) surface are clamped and nodes on the blue (right) surface are loaded with the measured reaction force. Symmetry conditions are applied.

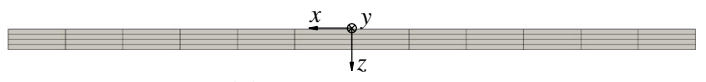

(b) Side view from left.

Fig. 6 Discretised FE model of the specimen. Tension direction aligns with 22 direction from the chosen material models, cf. (20), i.e. $M_{22}=M_{y y}=M_{\|}$. Reprinted from [22], with permission from Elsevier 
influence on the overall objective function value is ensured. Values vary for different identifications and are specified in Sects. 5.2 and 5.3. Displacement data in $z$-direction is not used and the related weights are also set to zero for all nodes. All identifications that involve temperature data are performed with a value of $W^{\theta}=1$.

Evaluation points are chosen to cluster around points, respectively loading states of significance, such as temperature maxima and minima or the onset of plasticity to enforce a better fit of the associated phenomena. The exact load steps at which the objective function is evaluated for each identification are indicated by markers in the associated stress-strain and temperature-time curves in Sects. 5.2 and 5.3. With the definitions above at hand, the two different experiments are subjected to a parameter identification.

\subsection{Experiment without cooling}

Data of the experiment without cooling is used for the following identification which is split into three parts. Results for model 1 and model 2 are presented side by side for each step of the process.

\subsubsection{Elastic material parameters}

The experimental reaction force of up to two seconds is prescribed as load to ensure that the elastic parameters are identified on the basis of purely elastic

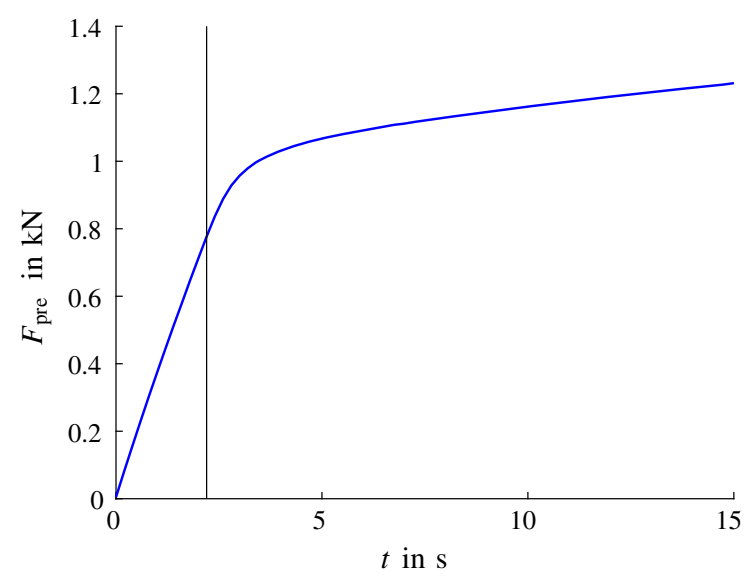

Fig. 7 Prescribed force $F_{\text {pre }}$ over time. Black, vertical line indicates end time $t_{\text {end }}^{\mathrm{el}}=2.0 \mathrm{~s}$ for identification of elastic parameters on the basis of the experiment without cooling experimental data, see Fig. 7. Only the error in displacements is considered for this part and the missing weighting factors are set to $W_{x}^{\mathrm{u}}=1$ and $W_{y}^{\mathrm{u}}=1$. The initial guess as well as the resulting material parameters of model 1 and model 2 are displayed in Table 3. The optimal elastic material parameters for model 1 and model 2 are identical, since both models share the same purely elastic material behaviour.

Regarding the quality of the fit, Fig. 9 exemplarily shows that the remaining error $f=f^{\mathrm{u}}$ is distributed rather homogeneously over the area which was considered for the identification. The distribution of the remaining deviation between experimental data and simulation results over time of a single, representative node can be seen in Fig. 8. The two Figures suggest a good fit of either model to the elastic material behaviour.

\subsubsection{Plastic material parameters}

With the elastic material parameters at hand, the plastic material parameters are identified in a second step by using experimental data up to the end of the experiment without cooling, i.e. up to $140 \mathrm{~s}$. The prescribed load path is displayed in Fig. 10.

Only the error in displacements is considered and the weighting factors are set to $W_{x}^{\mathrm{u}}=5$ and $W_{y}^{\mathrm{u}}=1$. The identification process is started with the initial guesses from Table 4 yielding the optimal material parameters displayed in the same Table.

Remaining deviations to the experimental data are sufficiently small as Figs. 11 and 12 indicate. Thus, the fit of the two models to the plastic material behaviour appears to be acceptably accurate.

Independent from the fit, a closer look at the obtained optimal plastic material parameters in Table 4 and the material response in Fig. 12 shows that the two models predict an almost identical

Table 3 Initial and obtained values for elastic material parameters on the basis of the experiment without cooling

\begin{tabular}{lll}
\hline Material parameter & $E[\mathrm{MPa}]$ & $v[-]$ \\
\hline Initial guess & 60000 & 0.30 \\
Optimal value-model 1 & 67913 & 0.35 \\
Optimal value-model 2 & 67913 & 0.35 \\
\hline
\end{tabular}


mechanical material response for the almost same set of material parameters. Thus, the assumption made in Remark 3, stating that the mechanical parts of the two models are comparable for large deformation, appears to be valid for the boundary value problem considered.

\subsubsection{Thermal material parameters}

Finally, the thermal material parameters of each model can be fitted to the temperature data of the experiment without cooling. The identification is started from several initial guesses, see Table 5, to investigate the uniqueness of the solution within a certain area of the parameter space, since a general statement about the correlation of the four thermal material parameters in Sect. 4.2 was not possible. Bounds for the parameter $\beta$ in model 2 are set in accordance with (26) to $0 \leq \beta \leq 6.92$. The predicted temperature evolution is almost identical for all optimised material parameter

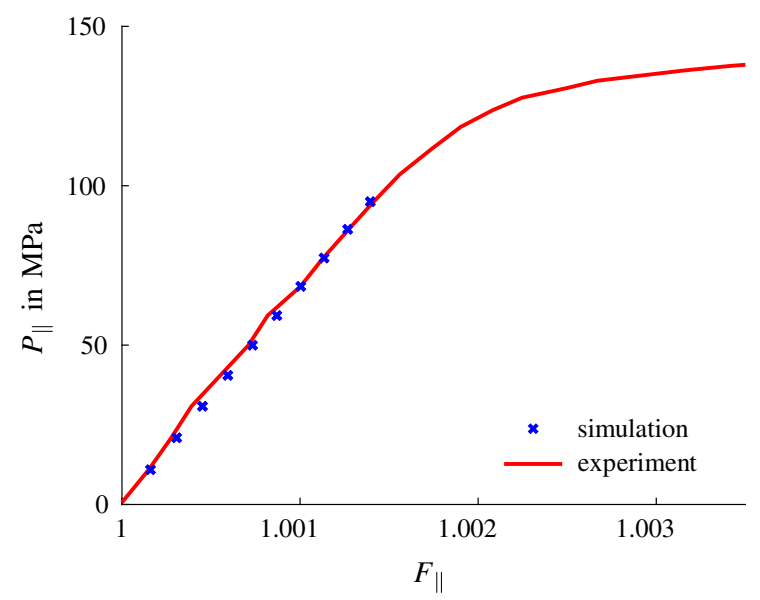

Fig. 8 Local stress-strain relation of experiment without cooling and of simulation with optimal elastic parameter set, evaluated at the midpoint of the specimen. Elastic response is identical for model 1 and model 2 sets, and remaining errors per node are rather small for both models as is indicated in Figs. 13 and 14.

Model 1 The optimal material parameter sets of model 1 differ significantly as can be seen in Table 6 . Thus, the inverse problem at hand has several solutions close to each other, and the assumption that a whole valley of solutions exists for the specific inverse problem at hand comes to mind. Either way, a strong correlation between scaling parameter $\beta$ and the remaining thermal material parameters becomes evident for this pair of model and experimental data.

Although a general statement was not possible in Sect. 4.2, the specific thermal boundary conditions for the BVP at hand eventually allow an interpretation of the correlation between $\beta, \alpha_{\exp }, \kappa_{\text {therm }}$ and $c_{0}$. It was already shown in [22], that the identification of thermal material parameters on the basis of an experiment without cooling, i.e. with monotonic loading, yields a unique (at least locally) result if a standard formulation of model 1 is used. The main

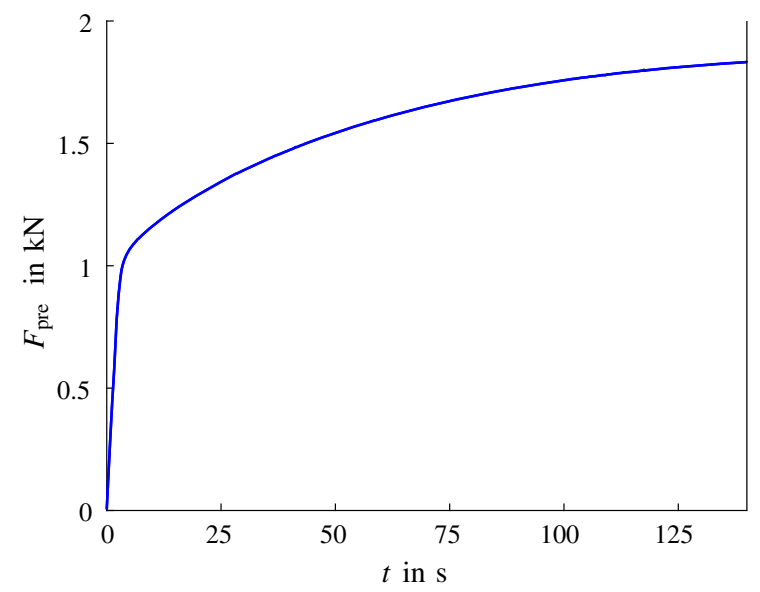

Fig. 10 Prescribed force $F_{\text {pre }}$ over time. Vertical, black line indicates end time $t_{\mathrm{end}}^{\mathrm{pl}}=140.0 \mathrm{~s}$ for identification of plastic parameters on the basis of the experiment without cooling
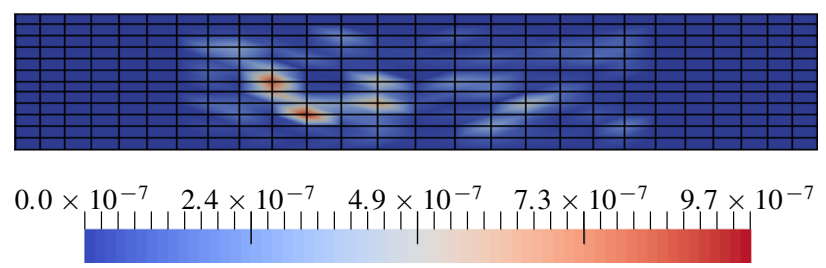

Fig. 9 Remaining nodal contribution to the mechanical (displacement) part of the objective function $f^{\mathrm{u}}$ at time $t=2.0 \mathrm{~s}$ using data from the experiment without cooling. The remaining error is the identical for model 1 and model 2 since the elastic response is identical 
Table 4 Initial and obtained values for plastic material parameters on the basis of the experiment without cooling

\begin{tabular}{lllll}
\hline Material parameter & $M_{y_{0}}[\mathrm{MPa}]$ & $M_{y_{\infty}}[\mathrm{MPa}]$ & $H_{\text {exp }}[-]$ & $F[-]$ \\
\hline Initial guess & 140.00 & 290.00 & 15.00 & 0.50 \\
Optimal value - Model 1 & 131.13 & 297.97 & 10.62 & 0.37 \\
Optimal value - Model 2 & 130.38 & 295.60 & 10.92 & 0.37 \\
\hline
\end{tabular}

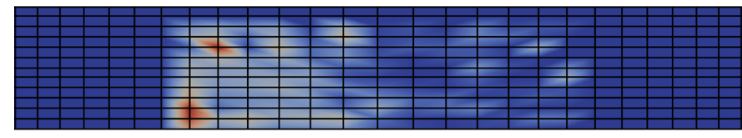

(a) Model 1.

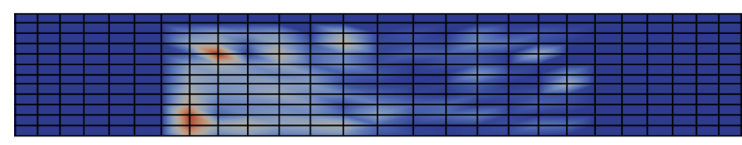

(b) Model 2 .

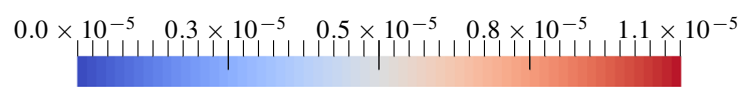

Fig. 11 Remaining nodal contribution to the mechanical (displacement) part of the objective function $f^{\mathrm{u}}$ at time $t=$ $140.0 \mathrm{~s}$ using data from the experiment without cooling

difference between such a standard formulation and the non-standard formulation used within this work is the parameter $\beta$. Whereas the dissipation of a standard model only depends on the mechanical material parameters, the amount of dissipated energy can still be scaled for the non-standard model without affecting the mechanical response, as was explained in Sect. 4. That is to say that $\beta$ affects the dissipation of all time steps alike by simply shifting the quantity of dissipated energy as can also be seen in Fig. 15. This constant difference in available heat is simply compensated by an adaptation of the other thermal material parameters. The following example is considered:If twice as much energy is transformed to heat at each time step, the heat capacity must be twice as high to ensure that the same (optimal) temperature is reached. The thermal conduction must be twice as high in order to transport twice as much heat towards both ends of the specimen where it can leave the specimen (due to the specific thermal boundary conditions at hand). By considering the balance of energy in (21), it follows that the heat expansion coefficient, which is featured in the addend responsible for the Gough-Joule effect, must also be twice as high for such a case. Accordingly, the ratio of

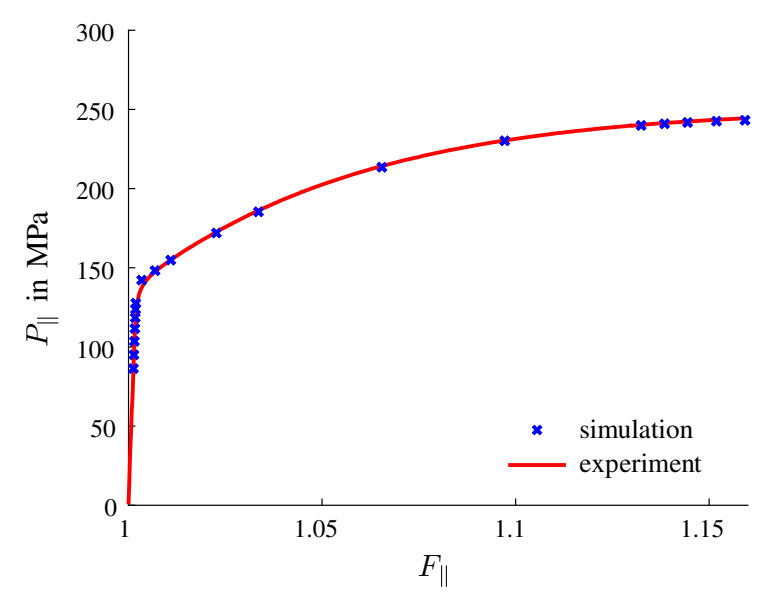

(a) Model 1 .

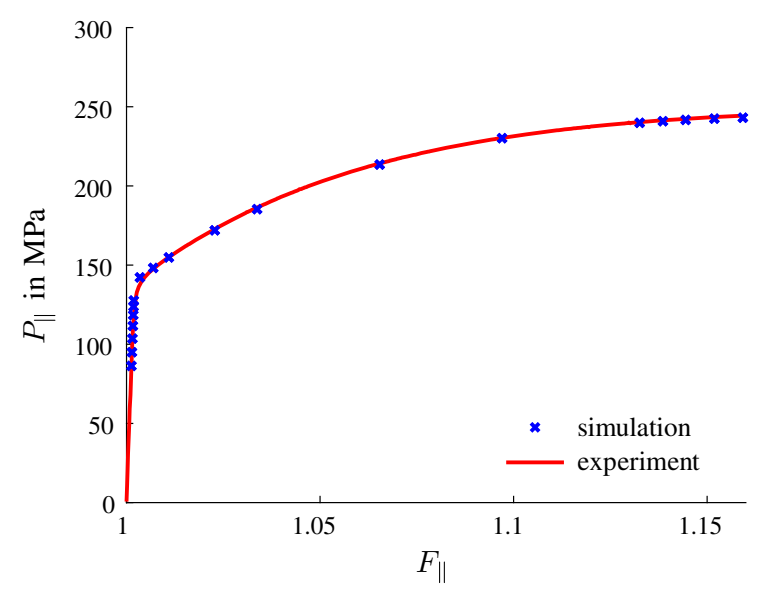

(b) Model 2 .

Fig. 12 Local stress-strain relation of experiment without cooling and of simulation with optimal plastic parameter set, evaluated at the midpoint of the specimen

$\alpha_{\exp } / \beta, \kappa_{\text {therm }} / \beta$ and $c_{0} / \beta$ should be constant if the amount of dissipated energy scales linearly in $\beta$. This is the case for model 1 and the results underline the reasoning above.

Apart from the correlation of the parameters, it is not possible to retrieve a thermal material parameter 
Table 5 Initial values for thermal material parameters for model 1 and model 2 and literature values

\begin{tabular}{lllll}
\hline Material parameter & $\alpha_{\exp }\left[10^{-6} / \mathrm{K}\right]$ & $\kappa_{\text {therm }}[\mathrm{mW} / \mathrm{mm} \mathrm{K}]$ & $c_{0}\left[\mathrm{~mJ} / \mathrm{mm}^{3} \mathrm{~K}\right]$ & $\beta[-]$ \\
\hline Initial guess $\boldsymbol{\kappa}_{\mathrm{A}}$ & 23.00 & 200.00 & 2.43 & 1.00 \\
Initial guess $\boldsymbol{\kappa}_{\mathrm{B}}$ & 23.00 & 200.00 & 2.43 & 0.10 \\
Initial guess $\boldsymbol{\kappa}_{\mathrm{C}}$ & 23.00 & 200.00 & 2.43 & 0.50 \\
Initial guess $\boldsymbol{\kappa}_{\mathrm{D}}$ & 12.00 & 400.00 & 1.22 & 0.50 \\
Literature value & 23.00 & 200.00 & 2.43 & - \\
\hline
\end{tabular}

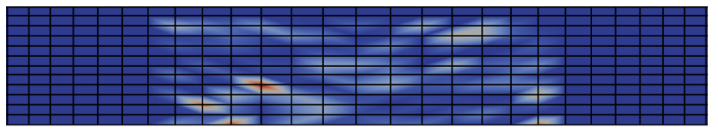

(a) Model 1. Distribution is almost identical for all optimal parameter sets.

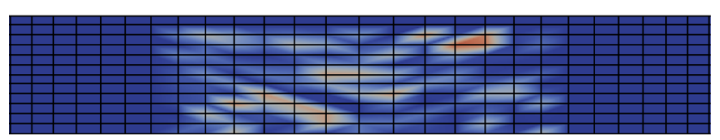

(b) Model 2 .

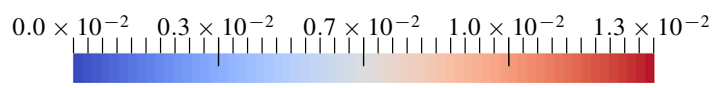

Fig. 13 Remaining nodal contribution to the thermal (temperature) part of the objective function $f^{\theta}$ at time $t=140.0 \mathrm{~s}$ using data of the experiment without cooling

set for model 1 which is close to the literature values, see Table 5. Thus, monotonous loading (a standard experiment in literature) does not hold enough information for an acceptable fit of this model which can be used to predict plastic heating as well as cooling due to thermal conduction.

Model 2 All obtained solutions for model 2 are numerically identical, see Table 7 , suggesting that a (locally) unique optimum has been found and that no correlation between the thermal parameters exists. This appears reasonable due to the difference to model 1 regarding the way $\beta$ affects the dissipation. The resulting format of the dissipation, see Equations 22 and 23 , shows that $\beta$ leads to a constant shift of dissipation for model 1 , but scales the dissipation load state dependent for model 2 . Thus, if only a particular functional relation between strain and dissipation is favourable regarding the plastic heating of the specimen, only one optimum exists.

The values of the optimal set, however, do also not resemble the literature values in Table 5 and it can be deduced that an experiment with monotonous loading alone does not hold enough information for an

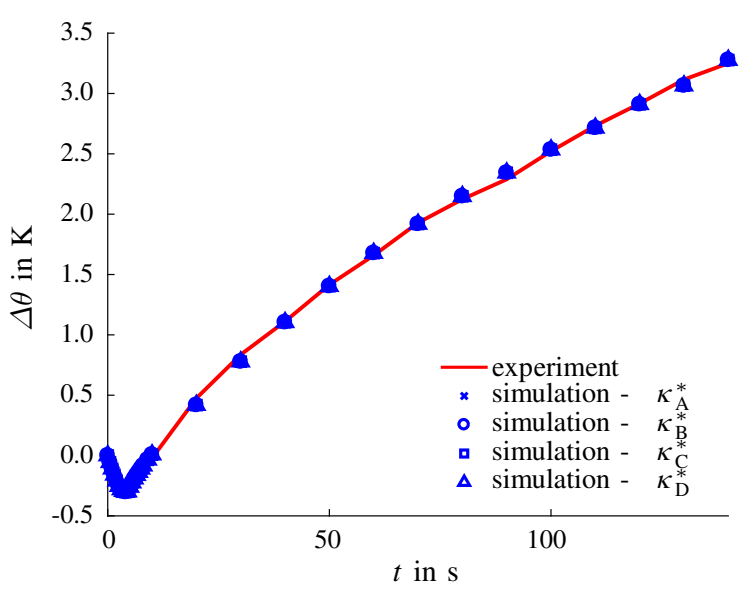

(a) Model 1 .

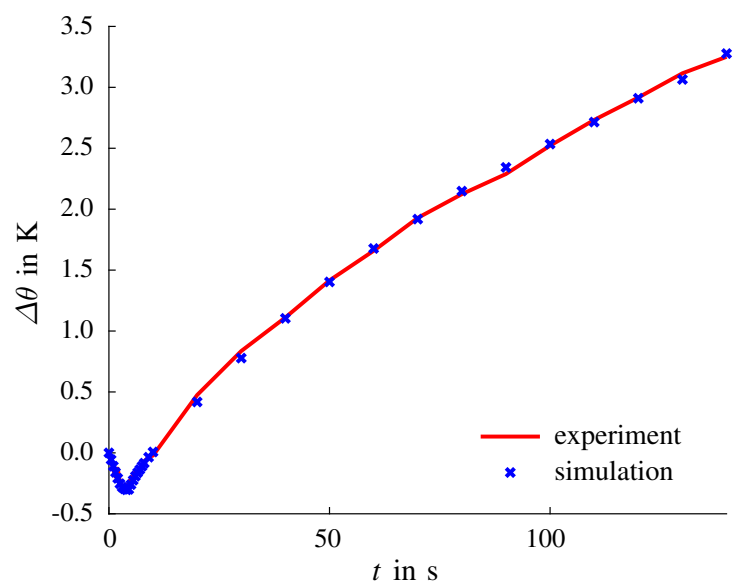

(b) Model 2 .

Fig. 14 Local temperature-time relation of experiment without cooling and of simulations with optimal thermal parameter sets, evaluated at the midpoint of the specimen

acceptable fit of model 2 which can be used to predict plastic heating as well as cooling due to thermal conduction. 
Table 6 Obtained values for thermal material parameters and remaining error contribution to the objective function $f^{\theta}$ for model 1 on the basis of the experiment without cooling

\begin{tabular}{llllll}
\hline Material parameter & $\alpha_{\exp }\left[10^{-6} / \mathrm{K}\right]$ & $\kappa_{\text {therm }}[\mathrm{mW} / \mathrm{mm} \mathrm{K}]$ & $c_{0}\left[\mathrm{~mJ} / \mathrm{mm}^{3} \mathrm{~K}\right]$ & $\beta[-]$ & $f^{\theta}[-]$ \\
\hline Optimal value $\boldsymbol{\kappa}_{\mathrm{A}}^{*}$ & 28.74 & 99.32 & 3.68 & 1.13 & 8.76 \\
Optimal value $\boldsymbol{\kappa}_{\mathrm{B}}^{*}$ & 37.06 & 128.15 & 4.68 & 1.46 & 8.76 \\
Optimal guess $\boldsymbol{\kappa}_{\mathrm{C}}^{*}$ & 30.57 & 105.65 & 3.90 & 1.21 & 8.76 \\
Optimal guess $\boldsymbol{\kappa}_{\mathrm{D}}^{*}$ & 18.97 & 65.49 & 2.46 & 0.75 & 8.76 \\
\hline
\end{tabular}

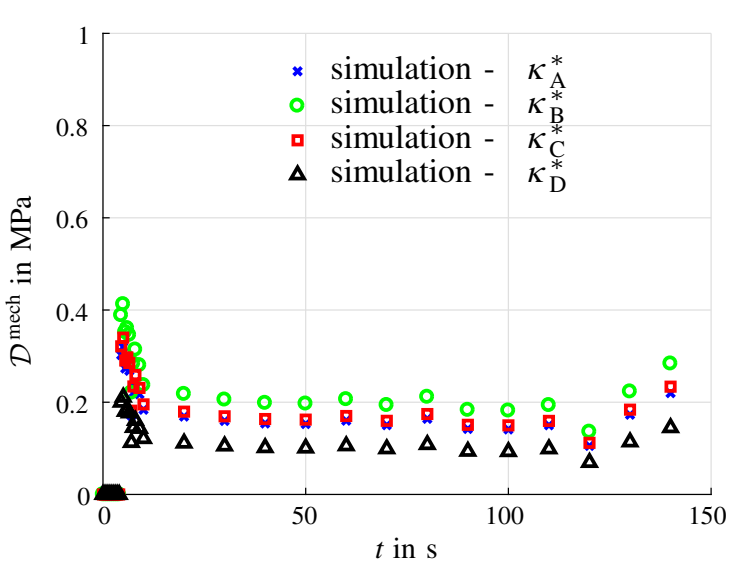

(a) Model 1 .

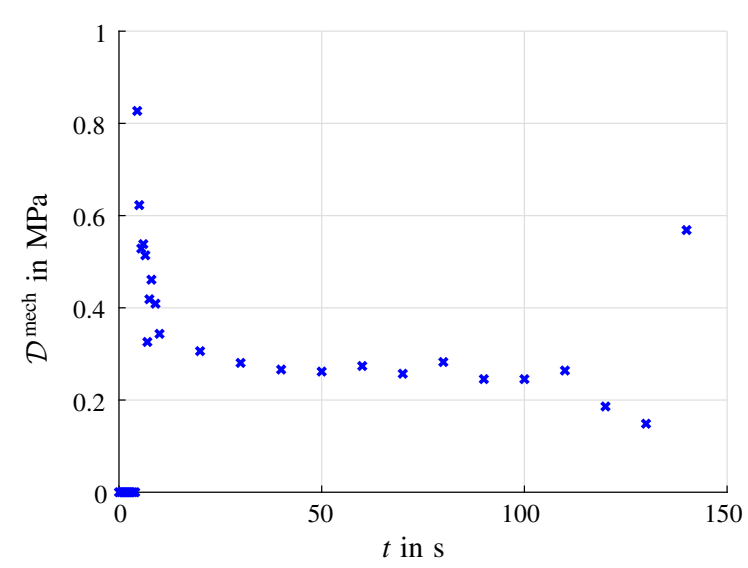

(b) Model 2 .

Fig. 15 Predicted local dissipation contribution during simulations of the experiment without cooling with optimal thermal parameter sets evaluated at midpoint of the specimen and at output time steps (subset of all time steps). Amount of dissipated energy density depends on applied load, i.e. the difference of applied force between two load steps. Due to the noise in the prescribed, experimental reaction force, some time steps have a larger or smaller increase in force than an ideal, smooth load path would generate. This leads to jumps in the dissipated energy density, which would otherwise represent a more smooth function for the case at hand

\subsection{Experiment with cooling}

Data of the experiment which includes a stage of cooling down to ambient temperature is used for the following identification. Results for model 1 and model 2 are presented side by side for each step of the identification process.

\subsubsection{Elastic material parameters}

The experimental reaction force of up to two seconds is prescribed as load to ensure that the elastic parameters are identified on the basis of purely elastic experimental data, see Fig. 16.

Only the error in displacements $f=f^{\mathrm{u}}$ is considered for this part and the missing weighting factors are set to $W_{x}^{\mathrm{u}}=1$ and $W_{y}^{\mathrm{u}}=1$. Initial guess as well as the resulting material parameters of model 1 and model 2 are displayed in Table 8 . The optimal elastic material parameters for model 1 and model 2 are again identical, since both models share the same purely elastic material behaviour.

Figure 18 shows that the remaining error $f=f^{\mathrm{u}}$ is distributed rather homogeneously over the area which was considered for the identification, similar to the identification on the basis of the experiment without cooling. The distribution of the remaining error over time of a single, representative node can be seen in Fig. 17. Both figures suggest a good fit of either model to the elastic material behaviour of the experiment with cooling.

\subsubsection{Plastic material parameters}

The plastic parameters are identified on the basis of experimental data up to $144 \mathrm{~s}$, taking into account the unloading of the specimen. Figure 19 shows the prescribed load path of the experimental reaction 
Table 7 Obtained values for thermal material parameters and remaining error contribution to the objective function $f^{\theta}$ for model 2 on the basis of the experiment without cooling

\begin{tabular}{llllll}
\hline Material parameter & $\alpha_{\exp }\left[10^{-6} / \mathrm{K}\right]$ & $\kappa_{\text {therm }}[\mathrm{mW} / \mathrm{mm} \mathrm{K}]$ & $c_{0}\left[\mathrm{~mJ} / \mathrm{mm}^{3} \mathrm{~K}\right]$ & $\beta[-]$ & $f^{\theta}[-]$ \\
\hline Optimal value $\boldsymbol{\kappa}_{\mathrm{A}}^{*}$ & 53.74 & 166.53 & 6.31 & 2.29 & 8.63 \\
Optimal value $\boldsymbol{\kappa}_{\mathrm{B}}^{*}$ & 53.74 & 166.53 & 6.31 & 2.29 & 8.63 \\
Optimal guess $\boldsymbol{\kappa}_{\mathrm{C}}^{*}$ & 53.74 & 166.53 & 6.31 & 2.29 & 8.63 \\
Optimal guess $\boldsymbol{\kappa}_{\mathrm{D}}^{*}$ & 53.74 & 166.53 & 6.31 & 2.29 & 8.63 \\
\hline
\end{tabular}

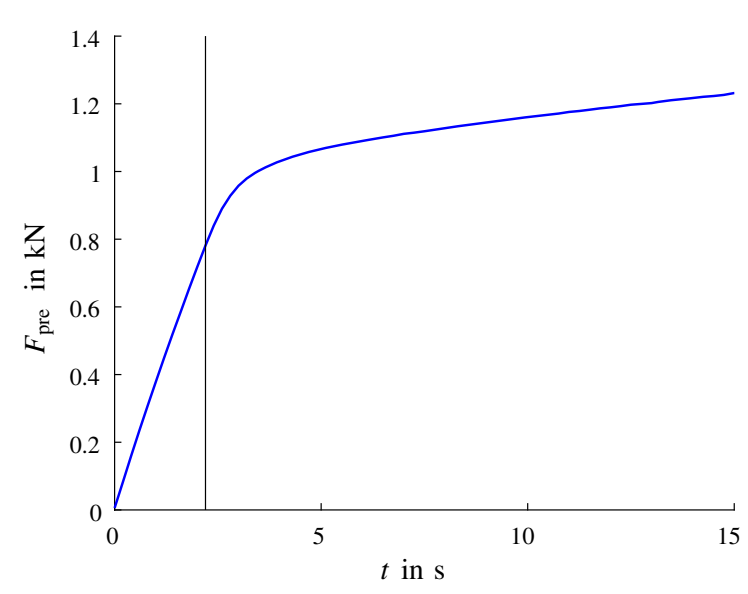

Fig. 16 Prescribed force $F_{\text {pre }}$ over time. Vertical, black line indicates end time $t_{\text {end }}^{\mathrm{el}}=2.0 \mathrm{~s}$ for identification of elastic parameters on the basis of the experiment with cooling

Table 8 Initial and obtained values for elastic material parameters on the basis of the experiment with cooling

\begin{tabular}{lll}
\hline Material parameter & $E[\mathrm{MPa}]$ & $v[-]$ \\
\hline Initial guess & 60000 & 0.30 \\
Optimal value-model 1 & 67026 & 0.36 \\
Optimal value-model 2 & 67026 & 0.36 \\
\hline
\end{tabular}

force. Only the error in displacements is considered and the weighting factors are set to $W_{x}^{\mathrm{u}}=5$ and $W_{y}^{\mathrm{u}}=1$.

Starting from the parameter sets identified on the basis of the experiment without cooling, the identification yields the optimised parameters depicted in Table 9.

Remaining deviations from the experimental data are sufficiently small as Figs. 20 and 21 indicate. Thus, both models have an almost identical and accurate fit to the elasto-plastic material behaviour, underlining once again the similarities of the two

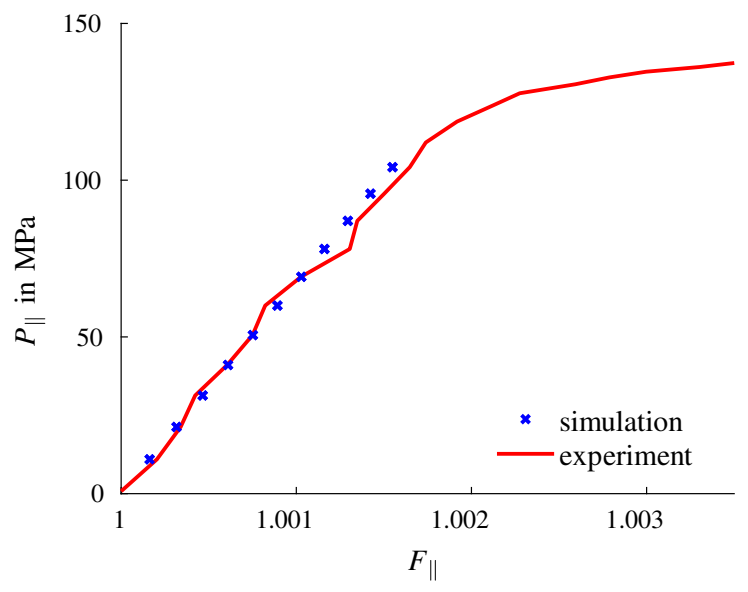

Fig. 17 Local stress-strain relation of experiment with cooling and of simulation with optimal elastic parameter set, evaluated at the midpoint of the specimen. Elastic response is identical for model 1 and model 2

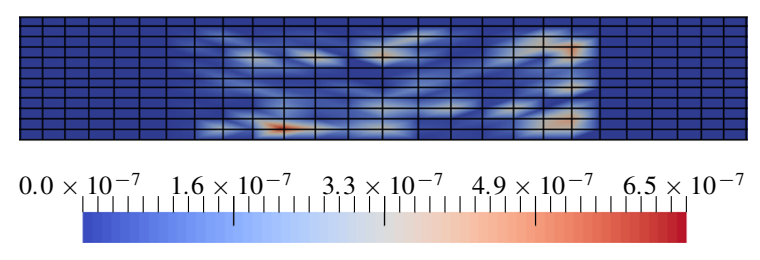

Fig. 18 Remaining nodal contribution to the mechanical (displacement) part of the objective function $f^{\mathrm{u}}$ at time $t=2.0$ $\mathrm{s}$ using data of the experiment with cooling. The remaining error is the same for model 1 and model 2 since the elastic response is identical

chosen model formulations with respect to the mechanical part.

\subsubsection{Thermal material parameters}

Finally, the thermal material parameters of each model are fitted to the temperature data of the experiment with cooling. The initial guesses from Table 5 are used 


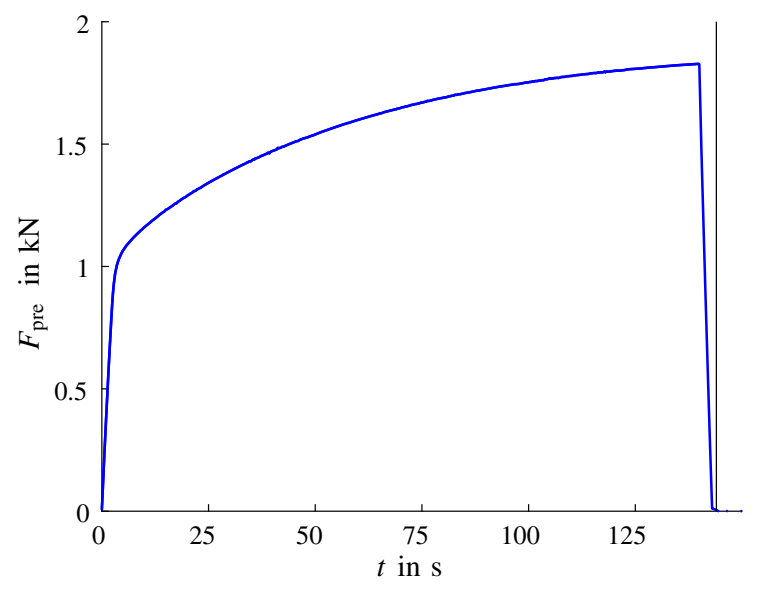

Fig. 19 Prescribed force $F_{\text {pre }}$ over time. Vertical, black line indicates end time $t_{\mathrm{end}}^{\mathrm{pl}}=144.0 \mathrm{~s}$ for identification of plastic parameters on the basis of the experiment with cooling

again to see whether the strong correlation between the four thermal material parameters of model 1 shown in Sect. 5.2.3 vanishes for the enhanced set of experimental data which includes the cooling of the specimen. The rough grit search approach furthermore shows whether it is possible to retrieve a parameter set that is sufficiently close to literature values. Bounds for the parameter $\beta$ in model 2 are set in accordance with (26) to $0 \leq \beta \leq 6.93$.

Model 1 All of the performed identifications converge towards the same optimum, see Table 10 . Thus, the additional information of the cooling process apparently stabilised the identification process for this model, removing the correlation described in Sect. 5.2.3. The resulting, optimal material response of model 1, however, still exhibits large remaining errors, see Fig. 22. Even though the remaining deviation from experimental data is distributed rather homogeneously in space, the comparison of experimental data and the optimal thermal material response over time in Fig. 24 reveals a substantial mismatch. It therefore appears impossible to predict the heating as well as the

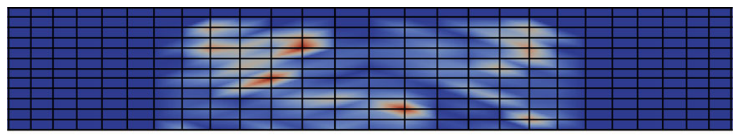

(a) Model 1 .

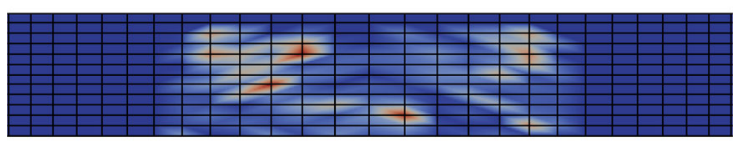

(b) Model 2 .

$0.0 \times 10^{-6} 0.8 \times 10^{-6} 1.6 \times 10^{-6} 2.4 \times 10^{-6} 3.1 \times 10^{-6}$

Fig. 20 Remaining nodal contribution to the mechanical (displacement) part of the objective function $f^{\mathrm{u}}$ at time $t=$ $140.0 \mathrm{~s}$ using data of the experiment with cooling

cooling of a specimen adequately with the associated plasticity model and a single set of material parameters within the range of temperature changes considered.

Model 2 All initial guesses lead to the same optimal set of thermal material parameters, indicating that a (locally) unique optimum is obtained for the case at hand. The remaining error per node, as exemplarily depicted in Fig. 23, is again rather low and statistically distributed. A closer look at the temperature evolution over time of a representative, single node in Fig. 24 reveals a very precise fit of computed to experimental data. Thus, model 2 appears to be capable of predicting plastic heating similarly well a thermal cooling effects with a single set of material parameters. Moreover, this optimal set is very close to the literature values in Table 5 with a relative deviation of

$$
\begin{aligned}
& \frac{\alpha_{\text {exp }}^{\text {lit }}-\alpha_{\text {exp }}^{\text {opt }}}{\alpha_{\text {exp }}^{\text {lit }}}=13.78 \%, \\
& \frac{\kappa_{\text {therm }}^{\text {lit }}-\kappa_{\text {therm }}^{\text {opt }}}{\kappa_{\text {therm }}^{\text {lit }}}=5.06 \%,
\end{aligned}
$$

Table 9 Initial and obtained values for plastic material parameters on the basis of the experiment with cooling

\begin{tabular}{lllll}
\hline Material parameter & $M_{y_{0}}[\mathrm{MPa}]$ & $M_{y_{\infty}}[\mathrm{MPa}]$ & $H_{\exp }[-]$ & 10.62 \\
\hline Initial guess & 131.13 & 297.97 & 10.82 & 0.37 \\
Optimal value-Model 1 & 129.27 & 293.38 & 10.97 & 0.37 \\
Optimal value-Model 2 & 129.28 & 293.36 & 0.37 \\
\hline
\end{tabular}




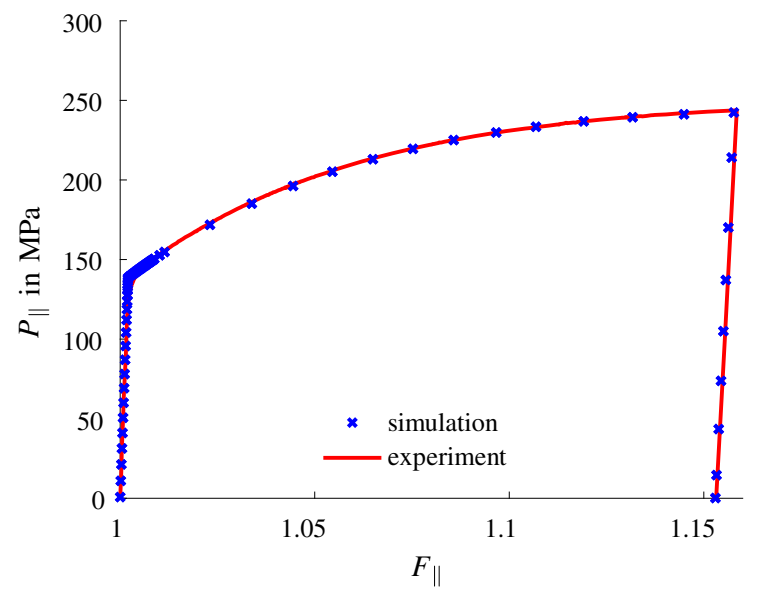

(a) Model 1.

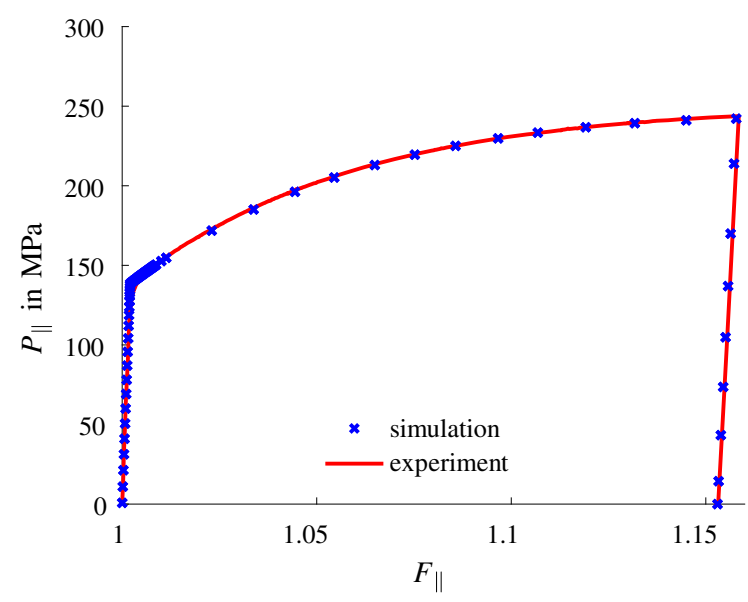

(b) Model 2 .

Fig. 21 Local stress-strain relation of the experiment with cooling and of simulation with optimal plastic parameter set, evaluated at the midpoint of the specimen

$\frac{c_{0}^{\mathrm{lit}}-c_{0}^{\mathrm{opt}}}{c_{0}^{\mathrm{lit}}}=9.05 \%$

which is not too uncommon for material parameters. The elastic parameters $E$ and $v$, for example, exhibit a
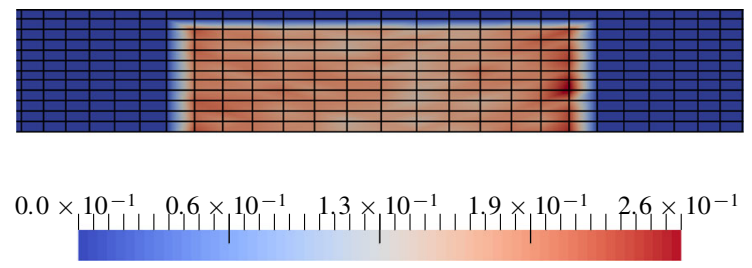

Fig. 22 Remaining nodal contribution to the thermal (temperature) part of the objective function $f^{\theta}$ at time $t=140.0 \mathrm{~s}$ using data of the experiment with cooling and material model 1

relative deviation of $5 \%$ and $9 \%$ with respect to literature values for aluminium.

It may appear odd that a similar set has not already been found for the monotonous loading case in Sect. 5.2 since both parameter sets lead to an apparently equally good fit of the heating phase. A comparison of the remaining error as described by the objective function value $f^{\theta}$ would give a closer insight to the quality of the fit but is not valid since the number of time steps is not equal. Thus, the error function is evaluated anew, comparing data from the experiment including cooling with the computed data of two simulations which both end at $140 \mathrm{~s}$, just prior to the unloading. One simulation uses the optimal thermal parameters obtained in 5.2.3 resulting in an remaining error of $f^{\theta}=5.16$. The second simulation uses the optimal thermal parameters from this section leading to a remaining error of $f^{\theta}=12.85$. It shows that the parameter set of this section may be able to describe heating and cooling alike. At the trade-off, however, that a slightly less perfect fit may be obtained than might be possible if only one phenomenon was considered at a time. The trade-off itself is very small and the fit nevertheless still very accurate as the previously mentioned Figures show, so that an accurate identification of the thermal parameters is possible with model 2 in combination with an experiment with cooling.

Table 10 Obtained values for thermal material parameters and remaining error contribution to the objective function $f^{\theta}$ for model 1 on the basis of the experiment with cooling

\begin{tabular}{llllll}
\hline Material parameter & $\alpha_{\exp }\left[10^{-6} / \mathrm{K}\right]$ & $\kappa_{\text {therm }}[\mathrm{mW} / \mathrm{mm} \mathrm{K}]$ & $c_{0}\left[\mathrm{~mJ} / \mathrm{mm}^{3} \mathrm{~K}\right]$ & $\beta[-]$ & $f^{\theta}[-]$ \\
\hline Optimal value $\boldsymbol{\kappa}_{\mathrm{A}}^{*}$ & 421.56 & 2653.29 & $\ll 1$ & 18.92 & 211.63 \\
Optimal value $\boldsymbol{\kappa}_{\mathrm{B}}^{*}$ & 421.40 & 2652.13 & $\ll 1$ & 18.91 & 211.63 \\
Optimal guess $\boldsymbol{\kappa}_{\mathrm{C}}^{*}$ & 421.35 & 2651.07 & $\ll 1$ & 18.90 & 211.63 \\
Optimal guess $\boldsymbol{\kappa}_{\mathrm{D}}^{*}$ & 421.47 & 2652.10 & $\ll 1$ & 18.91 & 211.63 \\
\hline
\end{tabular}




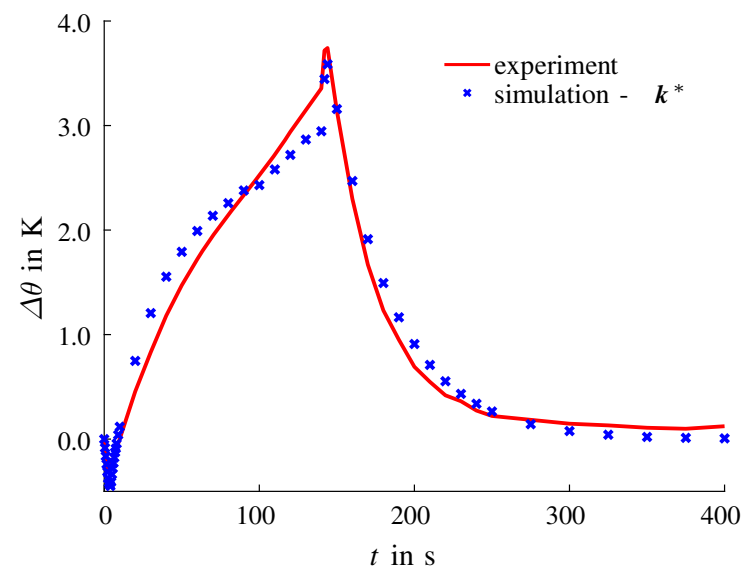

(a) Model 1 .

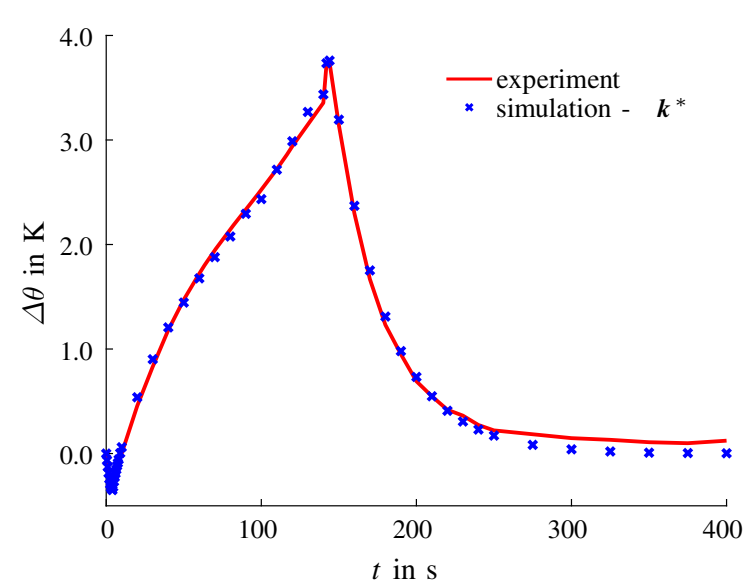

(b) Model 2.

Fig. 24 Local temperature-time relation of experiment with cooling and of simulations with optimal thermal parameter set, evaluated at the midpoint of the specimen
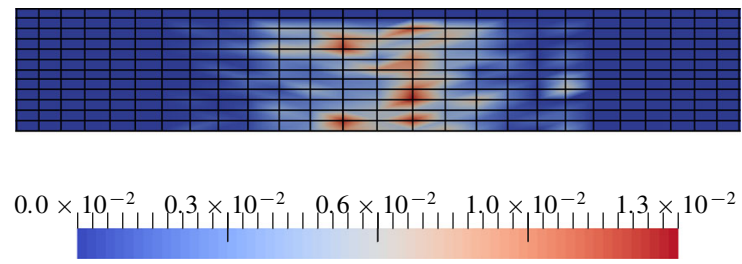

Fig. 23 Remaining nodal contribution to the thermal (temperature) part of the objective function $f^{\theta}$ at time $t=140.0 \mathrm{~s}$ using data of the experiment with cooling and material model 2

\section{Summary}

The material model formulation can have a large impact on the outcome of a parameter identification, especially with respect to the optimal values of the thermal material parameters. This issue is more closely investigated in this follow-up work to [22], where the framework for a parameter identification based on real, full field displacement and temperature data was first introduced. Focus of the work at hand lies on the influence of the predicted dissipation. Two classic material models, associated and non-associated plasticity, are extended to allow a thermo-mechanically consistent scaling of the dissipation by means of an additional material parameter. The dissipation itself follows directly from the postulated potentials and is a function of the current load state, requiring no further assumptions, e.g. regarding the relation towards cold work. It is shown that, for the low rise in temperature considered in this work, the two models predict the same mechanical response and differ only in the prediction of dissipated energy. The two models at hand are then subjected to two optimisation processes each, investigating whether it is possible to obtain a unique thermal parameter set which describes heating as well as cooling and analysing as to what kind of data is required to do so. All identifications make use of the small increase in temperature, separating the optimisation of mechanical and thermal material parameters.

At first, the data of an experiment with monotonous loading is used as the basis of an identification. The correlation in model 1 between the scaling parameter $\beta$ and the remaining thermal parameters leads to a whole valley of solutions for that model. A (locally) unique solution is obtained for model 2, due to the different evolution of dissipation. Both models fit the experimental data very well but neither parameter set is able to reliably predict the cooling of the specimen. Thus, data of an experiment with monotonous loading, a standard experiment in literature, does not allow an acceptable fit of the two standard models, meaning that the obtained thermal material parameters cannot be used for the computation of different (thermal) load scenarios.

In a second step, the data of an experiment with loading, unloading and cooling of the specimen is used as the basis of an identification. The fit of the mechanical field is again very precise. Regarding the optimisation of the thermal parameters for that second BVP, the results show that the afore mentioned correlation between the thermal parameters vanishes. Model 1, however, appears unable to represent both phenomena, plastic heating and cooling of the experimental temperature data at hand, resulting in an 
Table 11 Obtained values for thermal material parameters and remaining error contribution to the objective function $f^{\theta}$ for model 2 on the basis of the experiment with cooling

\begin{tabular}{llllll}
\hline Material parameter & $\alpha_{\exp }\left[10^{-6} / \mathrm{K}\right]$ & $\kappa_{\text {therm }}[\mathrm{mW} / \mathrm{mm} \mathrm{K}]$ & $c_{0}\left[\mathrm{~mJ} / \mathrm{mm}^{3} \mathrm{~K}\right]$ & $\beta[-]$ & $f^{\theta}[-]$ \\
\hline Optimal value $\boldsymbol{\kappa}_{\mathrm{A}}^{*}$ & 19.83 & 189.89 & 2.21 & 0.81 & 26.73 \\
Optimal value $\boldsymbol{\kappa}_{\mathrm{B}}^{*}$ & 19.83 & 189.89 & 2.21 & 0.81 & 26.73 \\
Optimal guess $\boldsymbol{\kappa}_{\mathrm{C}}^{*}$ & 19.83 & 189.89 & 2.21 & 0.81 & 26.73 \\
Optimal guess $\boldsymbol{\kappa}_{\mathrm{D}}^{*}$ & 19.83 & 189.89 & 2.21 & 0.81 & 26.73 \\
\hline
\end{tabular}

optimum with a large remaining error. In contrast to that, a unique solution can be obtained for model 2 which is also close to the literature values. Thus, even though the overall fit to the temperature data may not be as good as the fit to data for purely monotonic loading, model 2 is nevertheless able to represent plastic heating and cooling of the specimen almost equally well with a single set of material parameters. Model 1, however, can only represent either loading condition, necessitating different parameter sets for each type of loading, be it mechanical or purely thermal.

This work shows that even small changes in the model formulation can have a significant influence on the numerical value of the optimal material parameters and that a stable identification is possible, even if an additional unknown is added, i.e. a further material parameter which scales dissipation. While the results presented in [22] differed significantly from the literature values of the thermal material parameters, this work further emphasises that the framework presented therein can indeed be used to obtain such values, depending on the overall suitability of the chosen model. It underlines the statement that a material model should be carefully chosen when it comes to parameter identification for thermo-mechanically coupled material models. The influence of the predicted dissipation is non-neglectable. Not only does the resulting dissipation have an impact on the optimal value of the thermal material parameters, its evolution also determines what kind of data is required to avoid correlation between parameters and, even more importantly, whether or not a model is able to represent a certain BVP for a certain material accurately. It is worth noting that, although model 2 performs better for the material and load case considered in this work, it may perform entirely different for other materials. Regarding the uniqueness of the obtained optimum, the results show that experimental data of an experiment without cooling already holds, in principle, enough information for a locally unique solution of all thermal material parameters. This unique solution, however, can only be found if the model itself does not feature significant correlations between the material parameters considered.

In conclusion, a reliable identification of all material parameters, mechanical and thermal, of a thermomechanically coupled material model is possible if a careful choice regarding model and experimental data is made. Experimental information about the cooling down behaviour of the material significantly increases the overall fit of the thermal material parameters. Furthermore, the importance of validating material models is shown, in the sense that they are able to represent a certain load case for a specific material. Seeing that a common model such as model 1 (associated plasticity) is not able to represent plastic heating and cooling of the specimen equally well with a single set of material parameters, such a validation should probably be performed with every model before applying it to a more complex loading situation. Thus, the temperature evolution is either sound and optimal, or otherwise a different model should be chosen.

Funding Open Access funding enabled and organized by Projekt DEAL.. Partial financial support by the Deutsche Forschungsgemeinschaft (DFG, German Research Foundation) under Projektnummer 278868966, TRR 188, Project $\mathrm{C} 02$ is gratefully acknowledged.

\section{Compliance with ethical standards}

Conflict of interest The authors declare that they have no conflict of interest.

Open Access This article is licensed under a Creative Commons Attribution 4.0 International License, which 
permits use, sharing, adaptation, distribution and reproduction in any medium or format, as long as you give appropriate credit to the original author(s) and the source, provide a link to the Creative Commons licence, and indicate if changes were made. The images or other third party material in this article are included in the article's Creative Commons licence, unless indicated otherwise in a credit line to the material. If material is not included in the article's Creative Commons licence and your intended use is not permitted by statutory regulation or exceeds the permitted use, you will need to obtain permission directly from the copyright holder. To view a copy of this licence, visit http://creativecommons.org/licenses/by/4.0/.

\section{Appendix: A Limits for scaling parameter $\beta$ in model 2}

Boundaries for the material parameter $\beta$ are derived in this section to ensure that the dissipation of model 2 , with non-associated evolution equations, is always larger or equal to zero.

The dissipation of model 2 is given in (23) so that the associated dissipation inequality can be written as

$$
\mathcal{D}^{\mathrm{mech}}=\lambda_{\mathrm{pm}}\left[b h \alpha^{2}+[1-\beta] b M_{y_{0}} \alpha+\beta M_{y_{0}}\right] \geq 0 .
$$

For this model, the plastic multiplier will always be non-negative and we will assume that the same holds for the mechanical material parameters. Thus, inequality (33) is always fulfilled as long as

$$
\begin{array}{r}
D(\alpha)=b h \alpha^{2}+[1-\beta] b M_{y_{0}} \alpha+\beta M_{y_{0}} \geq 0, \text { with } \\
\lambda_{\mathrm{pm}} \geq 0 .
\end{array}
$$

Recalling that $b \geq 0$ and $h \geq 0$, this function can be identified to be a convex, quadratic function in $\alpha$ with a y-intercept of $D(\alpha=0)=\beta M_{y_{0}}$. It is now sufficient to ensure that

$$
D \geq 0 \quad \forall \alpha \geq 0
$$

since $\alpha$ evolves positive for the model at hand. A first, lower boundary for the parameter $\beta$ can easily be obtained by considering the y-intercept of the continuous function $D . D(\alpha=0) \geq 0$ has to be fulfilled in order to ensure that the particular point of $\alpha=0$ complies with (35). Hence

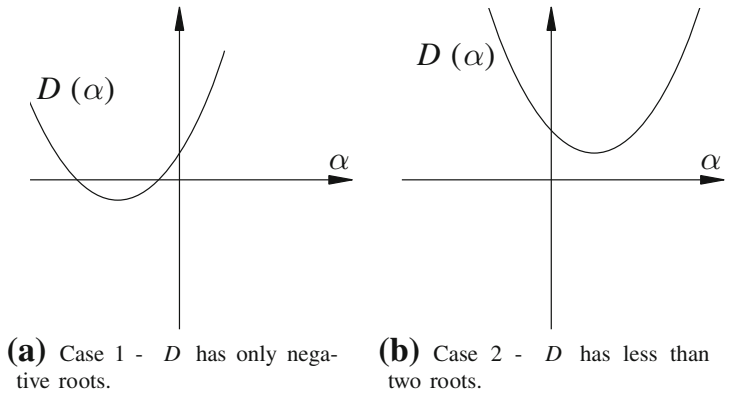

Fig. $25 D$ is a convex, quadratic function in $\alpha$ and required to be in the first quadrant for all $\alpha>0$ to ensure a dissipation which is larger or equal to zero

$$
\beta \geq 0
$$

must hold to ensure a positive y-intercept. Further conditions for $\beta$ can be derived by considering that the dissipation of the model will always be larger or equal to zero if the function

$$
\begin{aligned}
D(\alpha)=b h \alpha^{2}+[1-\beta] b M_{y_{0}} \alpha+\beta M_{y_{0}} & \geq 0, \quad \text { with } \\
\beta, b, h, M_{y_{0}} & \geq 0
\end{aligned}
$$

lies only in the first quadrant for $\alpha>0$. There are only two possibilities for a convex, quadratic function with a positive $y$-intercept fulfilling this condition. Either both roots of the function must be negative, see Fig. 25a, or there are less than two roots, see Fig. 25b. In summation, the dissipation of the model is guaranteed to be larger or equal to zero if $\beta$ is chosen such that either condition is met.

Case 1 -negative roots The two roots $\alpha_{1,2}$ of function $D$ can be computed by

$$
\alpha_{1,2}=-\frac{[1-\beta] M_{y_{0}}}{2 h} \pm\left|\sqrt{\frac{[1-\beta]^{2} M_{y_{0}}^{2}}{4 h^{2}}-\beta \frac{M_{y_{0}}}{h b}}\right| .
$$

It has already been established that $\beta \geq 0$ so that only two different cases must be considered.

1. $\beta \leq 1-$ The first addend is negative for this case. Hence, it suffices to enforce the larger of the two roots 
$\alpha_{1}=-\frac{[1-\beta] M_{y_{0}}}{2 h}+\left|\sqrt{\frac{[1-\beta]^{2} M_{y_{0}}^{2}}{4 h^{2}}-\beta \frac{M_{y_{0}}}{h b}}\right|$

to be negative, resulting in the inequality

$$
\begin{aligned}
\alpha_{1} & =-\frac{[1-\beta] M_{y_{0}}}{2 h}+\left|\sqrt{\frac{[1-\beta]^{2} M_{y_{0}}^{2}}{4 h^{2}}-\beta \frac{M_{y_{0}}}{h b}}\right| \leq 0 \\
& \Leftrightarrow \frac{[1-\beta] M_{y_{0}}}{2 h} \geq\left|\sqrt{\frac{[1-\beta]^{2} M_{y_{0}}^{2}}{4 h^{2}}-\beta \frac{M_{y_{0}}}{h b}}\right| \\
& \Leftrightarrow\left|\frac{[1-\beta] M_{y_{0}}}{2 h}\right| \geq\left|\sqrt{\frac{[1-\beta]^{2} M_{y_{0}}^{2}}{4 h^{2}}-\beta \frac{M_{y_{0}}}{h b}}\right| \\
& \text { with } 0 \leq \beta \leq 1 \\
& \Leftrightarrow \frac{[1-\beta]^{2} M_{y_{0}}^{2}}{4 h^{2}} \geq \frac{[1-\beta]^{2} M_{y_{0}}^{2}}{4 h^{2}}-\beta \frac{M_{y_{0}}}{h b} \\
& \Leftrightarrow \beta \geq 0 .
\end{aligned}
$$

This renders one set of bounds for the material parameter $\beta$ to be $0 \leq \beta \leq 1$.

2. $\beta>1$ - The first addend is positive for this case and it must again be ensured that the larger of the two roots $\alpha_{1}$ is negative. This however will not occur since

$$
\alpha_{1}=-\frac{[1-\beta] M_{y_{0}}}{2 h}+\left|\sqrt{\frac{[1-\beta]^{2} M_{y_{0}}^{2}}{4 h^{2}}-\beta \frac{M_{y_{0}}}{h b}}\right|
$$

is the sum of two positive addends. It is therefore impossible to have only negative roots if $\beta>1$ and the resulting bounds are again $0 \leq \beta \leq 1$.

The roots of function $D$ are therefore negative if $0 \leq \beta \leq 1$, leading to a dissipation of the model which is larger or equal to zero.

Case 2-less than two roots The quadratic function $D$ has less than two roots (in $\mathbb{R}$ ) in the case that the radicand of Equation (38) $r=\frac{[1-\beta]^{2} M_{y_{0}}^{2}}{4 h^{2}}-\beta \frac{M_{y_{0}}}{h b}$

is zero (only one root) or negative (no roots). This is the case if $\beta$ lies between the two roots of $r$

$\beta_{\mathrm{u}, 1}=\frac{M_{y_{0}} b+2 h\left[1 \pm \sqrt{\frac{M_{y_{0}} b}{h}+1}\right]}{M_{y_{0}} b}$

since $r$ is a convex, quadratic function in $\beta$. Following therefrom, the convex function $D$ has less than two roots in $\mathbb{R}$ so that the dissipation is always larger or equal to zero for

$\beta_{1} \leq \beta \leq \beta_{\mathrm{u}}$.

Hence, the total set of bounds for the material parameter $\beta$ which ensures a fulfilment of the second law of thermodynamics reads

$$
\begin{gathered}
0 \leq \beta \leq 1 \quad \vee \quad \beta_{1} \leq \beta \leq \beta_{\mathrm{u}} \text {, with } \\
\beta_{\mathrm{u}, 1}=\frac{M_{y_{0}} b+2 h\left[1 \pm \sqrt{\frac{M_{y_{0}} b}{h}+1}\right]}{M_{y_{0}} b} .
\end{gathered}
$$

\section{References}

1. Adamczyk WP, Kruczek T, Moskal G, Białecki RA (2017) Nondestructive technique of measuring heat conductivity of thermal barrier coatings. Int $\mathrm{J}$ Heat Mass Transf 111:442-450

2. Bartels A, Bartel T, Canadija M, Mosler J (2015) On the thermomechanical coupling in dissipative materials: a variational approach for generalized standard materials. J Mech Phys Solids 82:218-234

3. Berthelsen R, Tomath D, Denzer R, Menzel A (2016) Finite element simulation of coating-induced heat transfer: application to thermal spraying processes. Meccanica 51(2):291-307

4. Box M (1965) A new method of constrained optimization and a comparison with other methods. Comput J 8(1):42-52

5. Farren WS, Taylor GL (1925) The heat developed during plastic extension of metals. Proc R Soc Lon Ser A Contain Papers Math Phys Charact 107(743):422-451

6. Hartmann S, Gilbert RR (2018) Identifiability of material parameters in solid mechanics. Arch Appl Mech $88(1-2): 3-26$

7. Hodowany J, Ravichandran G, Rosakis A, Rosakis P (2000) Partition of plastic work into heat and stored energy in metals. Exp Mech 40(2):113-123 
8. Johnson SG (2014) The NLopt nonlinear-optimization package http://ab-initio.mit.edu/nlopt

9. Knysh P, Korkolis YP (2015) Determination of the fraction of plastic work converted into heat in metals. Mech Mater 86:71-80

10. Kruczek T, Adamczyk WP, Bialecki RA (2013) In situ measurement of thermal diffusivity in anisotropic media. Int J Thermophys 34(3):467-485

11. Louche H, Schlosser P, Favier D, Orgéas L (2012) Heat source processing for localized deformation with non-constant thermal conductivity. Application to superelastic tensile tests of NiTi shape memory alloys. Exp Mech 52(9):1313-1328

12. Macdougall D (2000) Determination of the plastic work converted to heat using radiometry. Exp Mech 40(3):298-306

13. Mahnken R (1996) Theoretische und numerische Aspekte zur Modellierung und Parameteridentifikation bei metallischen Werkstoffen. Universität Hannover, Habilitation

14. Mahnken R (2000) A comprehensive study of a multiplicative elastoplasticity model coupled to damage including parameter identification. Comput Struct 74(2):179-200

15. Mason J, Rosakis A, Ravichandran G (1994) On the strain and strain rate dependence of the fraction of plastic work converted to heat: an experimental study using high speed infrared detectors and the kolsky bar. Mech Mater 17(2-3):135-145

16. Nelder JA, Mead R (1965) A simplex method for function minimization. Comput J 7(4):308-313

17. Parker W, Jenkins R, Butler C, Abbott G (1961) Flash method of determining thermal diffusivity, heat capacity, and thermal conductivity. J Appl Phys 32(9):1679-1684
18. Pottier T, Toussaint F, Louche H, Vacher P (2013) Inelastic heat fraction estimation from two successive mechanical and thermal analyses and full-field measurements. Eur $\mathbf{J}$ Mech A Solids 38:1-11

19. Ristinmaa M, Wallin M, Ottosen NS (2007) Thermodynamic format and heat generation of isotropic hardening plasticity. Acta Mech 194(1-4):103-121

20. Rittel D (1999) On the conversion of plastic work to heat during high strain rate deformation of glassy polymers. Mech Mater 31(2):131-139

21. Rosakis P, Rosakis A, Ravichandran G, Hodowany J (2000) A thermodynamic internal variable model for the partition of plastic work into heat and stored energy in metals. J Mech Phys Solids 48(3):581-607

22. Rose L, Menzel A (2020) Optimisation based material parameter identification using full field displacement and temperature measurements. Mech Mater 145:103292

23. Stainier L, Ortiz M (2010) Study and validation of a variational theory of thermo-mechanical coupling in finite visco-plasticity. Int J Solids Struct 47(5):705-715

24. Taylor GI, Quinney H (1934) The latent energy remaining in a metal after cold working. Proc R Soc Lond Ser A Contain Papers Math Phys Charact 143(849):307-326

25. Yang Q, Stainier L, Ortiz M (2006) A variational formulation of the coupled thermo-mechanical boundary-value problem for general dissipative solids. J Mech Phys Solids 54(2):401-424

Publisher's Note Springer Nature remains neutral with regard to jurisdictional claims in published maps and institutional affiliations. 